\title{
The theory and application of eulerlets
}

Cite as: Phys. Fluids 31, 047106 (2019); https://doi.org/10.1063/1.5088132

Submitted: 08 January 2019 . Accepted: 02 April 2019 . Published Online: 23 April 2019

E. Chadwick (D), J. M. Christian, A. Kapoulas (D), and K. Chalasani
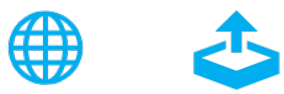

\section{CAPTURE WHAT'S POSSIBLE}

WITH OUR NEW PUBLISHING ACADEMY RESOURCES

Learn more $\Theta$

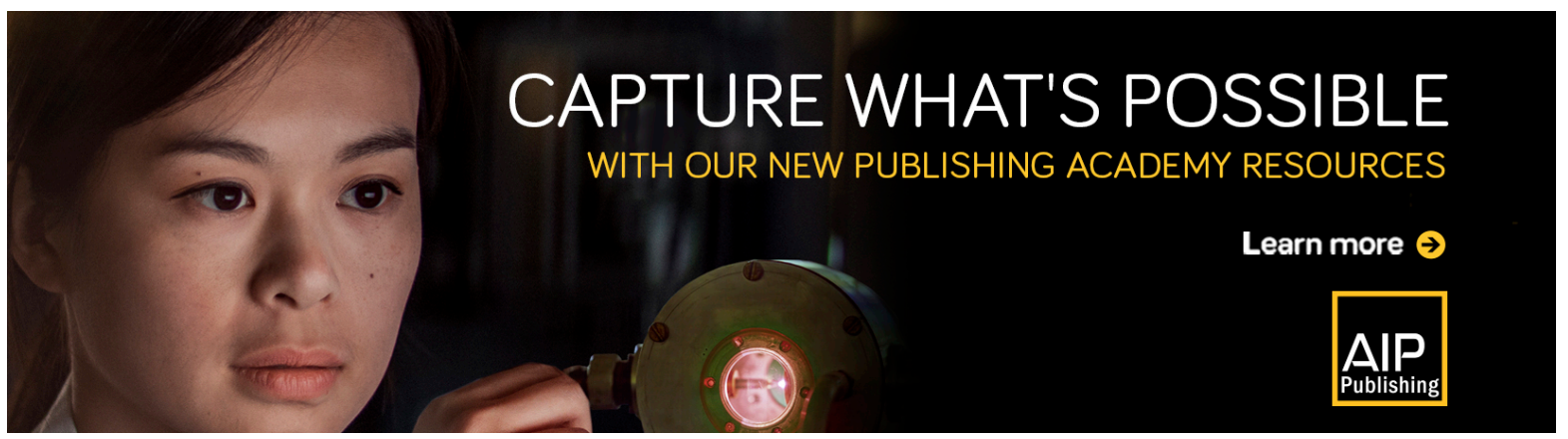




\title{
The theory and application of eulerlets
}

\author{
Cite as: Phys. Fluids 31, 047106 (2019); doi: 10.1063/1.5088132 \\ Submitted: 8 January 2019 • Accepted: 2 April 2019 • \\ Published Online: 23 April 2019
}

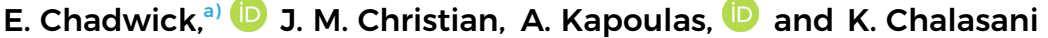

\author{
AFFILIATIONS \\ Mathematics, University of Salford, Salford M54WT, United Kingdom
}

a) Electronic mail: e.a.chadwick@salford.ac.uk

\begin{abstract}
Consider a fixed body in a uniform flow field in the limit as the Reynolds number approaches infinity and the flow field remains steady. Instead of using standard techniques and theory for describing the problem, a new method is employed based upon the concept of matching two different Green's integral representations over a common boundary, one given by approximations valid in the near-field and the other by approximations in the far-field. Further novelty arises from the choice of a near-field, that is, the Euler flow matched to an Oseen flow far-field. This entails introducing and defining eulerlets that are Green's functions of the Euler equation. One important consequence of the model is the presence of a new Euler wake velocity not captured in standard models. This has a constant unchanging downstream profile and arises from the matching to the far-field Oseen wake velocity. It is then shown how this representation reduces to classical inviscid ideal flow aerodynamics when applied to flow past aerofoils and wings. It is also shown how it reduces to slender body flow theory. Finally, the formulation is tested on uniform flow past a circular cylinder for mean-steady subcritical laminar flow and turbulent flow. The inviscid impermeability boundary condition is used, the drag coefficient is specified, and a constant distribution of drag eulerlets is modeled. The forward flow separation and pressure drop in the wake are captured and compare favorably with experiment. The future expectation is the modeling of multiple general shaped bodies.
\end{abstract}

Published under license by AIP Publishing. https://doi.org/10.1063/1.5088132

\section{NOMENCLATURE}

$\rho, \mu, U, l$

Dimensional parameter: density, coefficient of viscosity, uniform stream velocity, and typical body

$R e=\rho U l / \mu$

$k=\operatorname{Re} / 2$

$\varepsilon$

Superscript $\dagger$

$\mathbf{u}^{\dagger}, p^{\dagger}, \mathbf{x}^{\dagger}$

$\mathbf{u}, p$

$\mathbf{x}$

$\mathbf{x}^{\prime}$

Superscript $O$ Superscript $E$ Superscript $I$ Superscript $I I$ $\eta$ length (for the circular cylinder this is the radius)

Dimensionless Reynolds number

Dimensionless wake number

Order of perturbation to uniform flow field

Dimensional variable: Navier-Stokes

Velocity, pressure, and position vector

Dimensional Navier-Stokes velocity and pressure

perturbed to a uniform stream

Dimensionless position vector

Variable of integration in Green's integral of the

dimensionless position vector

Dimensionless Oseen variables

Dimensionless Euler variables

Leading order variable in Oseen perturbation

Second order variable in Oseen perturbation

The wake variable in two-dimensions

The wake variable in three-dimensions $r$

$r^{*}$

R

$a_{i}$

$a_{\alpha}$

$a_{, i}$

$a_{; i}$

$\phi$

$\mathbf{w}$

$\chi$

$\Sigma, d \Sigma$,

$\partial \Sigma$,

$\partial \Sigma_{M}$,

$d \sigma$

$\delta$

$\delta\left(x_{i}\right)$

$H\left(x_{1}\right)$

$\delta_{i j}$

$\varepsilon_{i j k}$
Dimensionless radial length in two dimensions

Cross-sectional dimensionless radial length $\sqrt{x_{2}^{2}+x_{3}^{2}}$ Dimensionless radial length in three dimensions Vector representation with English index starting from 1 and ending at dimension of space

Vector representation with Greek index starting from 2 and ending at dimension of space

Differentiation of $a$ with respect to $x_{i}$ Differentiation of $a$ with respect to $x_{i}^{\prime}$

Velocity potential satisfying the Laplace equation

Wake velocity

Wake velocity potential

A region of space, an element of the space,

The boundary of the region of space,

The matching boundary,

An element of the boundary

Dirac delta function of the variables of the space

Dirac delta function of the variable $x_{i}$

Heaviside function of the variable $x_{1}$

Kronecker delta

Three-dimensional Levi-Civita symbol 


\section{INTRODUCTION}

The Euler flow and, in particular, potential flow are important for designing maneuvering bodies in air and water, for example, the design of the shape of a wing or the hull of a boat. This is because it is understood how changes in the potential flow representation lead to changes in the maneuvering characteristics of the body. This is in contrast to computational fluid dynamics solvers where there is no representation in terms of mathematical functions, and instead, the solver computes directly the differential equation. In the present paper, it is proposed to introduce a new theory for the Euler flow that uses eulerlets, which we define as Green's functions in the Euler flow. The eulerlet Green's function is defined as the impulsive (Dirac delta function) response at the origin for the Euler equations in an unbounded fluid with a uniform flow field far from the origin. The response in the direction parallel to the flow field is called the drag eulerlet, and the responses perpendicular to the flow field are called the lift and side-force eulerlets. A pictorial representation of the drag and lift eulerlets are given in Figs. 1 and 2.

By matching to a far-field Oseen flow, it turns out that a Green's integral representation for the Euler velocity is possible. This is because we shall find in the present paper that in the high Reynolds number limit, the oseenlets tend to the eulerlets on the matching boundary. As well, the Euler equation and Oseen equation match. The main consequence of this theory is the existence of an Euler wake which has a profile over the cross-sectional plane perpendicular to the flow direction that remains unchanged at every downstream wake position. In particular, there is an Euler wake inflow giving drag.

This theory is the culmination of the work by Chadwick and co-workers on potential and Oseen flows; Chadwick presented the far-field Oseen velocity description for a fixed body in a uniform flow field. ${ }^{1}$ It was shown how the far-field velocity is represented by an integral distribution of oseenlets, as described by Oseen. ${ }^{2}$ This relies on certain far-field integral contributions tending to zero, as subsequently shown by Fishwick and Chadwick. ${ }^{3}$ This far-field velocity representation can then be matched to a near-field flow, such as the low Reynolds number Stokes flow. ${ }^{4}$ For the high Reynolds number

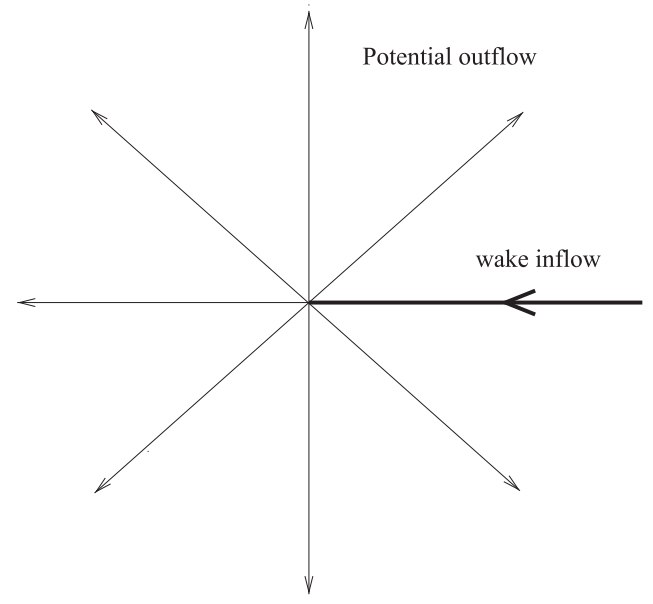

FIG. 1. Streamlines for the drag eulerlet.

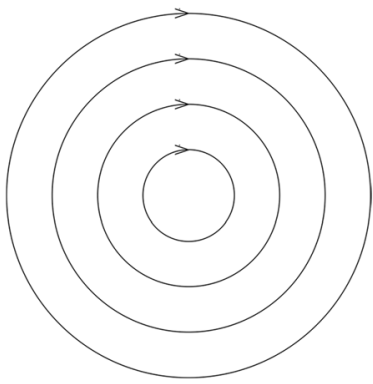

(a)

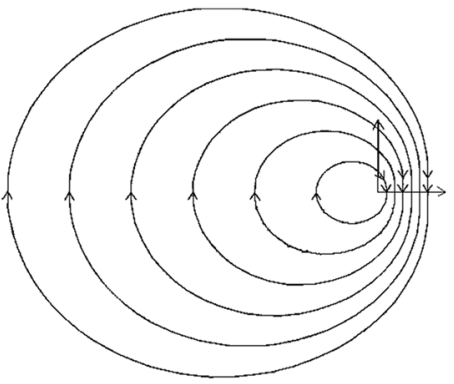

(b)
FIG. 2. Streamlines for the lift eulerlet. (a) Streamlines for the $2 \mathrm{D}$ lift eulerlet. (b) Streamlines for the 3D lift eulerlet.

steady flow, it can also be continued into the near-field giving an Oseen flow for slender-bodies. ${ }^{5}$ For this case, a discrepancy in the description of the wake is found between the Oseen model and the standard inviscid potential flow model. This gives rise to a difference between the two models for the calculation of the forces on the slender body. Subsequently, the experiment verified that the Oseen model, rather than the potential flow model, evaluated lift correctly for slender bodies of different elliptic cross section; ${ }^{6}$ see Fig. 3. Similarly, by comparing the lift oseenlet and the inviscid potential horseshoe vortex, a new slender wing potential flow model is obtained. ${ }^{7}$ This is different from standard potential flow theory in that it also includes a singular vortex-wake-velocity part originating from the lift oseenlet. This is the core of the Oseen vortex line. ${ }^{8}$ This difference also produces a lift discrepancy between existing Euler and Oseen flow models which leads to a proposal for an alternative model from the Oseen flow in the high Reynolds number limit. ${ }^{9}$ This suggests developing a new Euler model by considering eulerlets, which in Ref. 10 are obtained from oseenlets in the limit as the Reynolds number tends to infinity. In this case, the term that produces the difference and discrepancy within the eulerlet is a nonpotential core originating from the limiting value of the singular vortex-wake-velocity part that lies along a semi-infinite wake half-line.

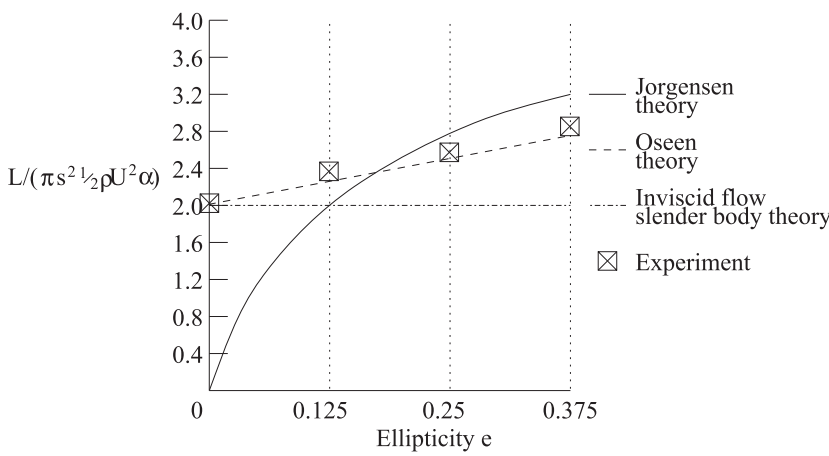

FIG. 3. Comparison of lift between Oseen theory, inviscid potential flow theory, and Jorgensen theory against the experiment for a slender body with the elliptic cross section. 
This allows us to also consider bluff body flows by an eulerlet model, ${ }^{11,12}$ where the problem of steady uniform flow past a circular cylinder is considered. However, steady flow breaks down above a Reynolds numbers of 40 , so the eulerlet model only approximately holds. Despite this, for a Reynolds number up to 40, good agreement is shown with the far-field wake profile and formation of wake eddies.

In the present paper, we focus on bringing together all this previous work within a general and overarching eulerlet theory that encompasses slender-body theory, lifting wing theory, and bluffbody flow. In particular, we shall assume a far-field Oseen flow field that is matched to a near-field Euler flow field. The matching is made by comparing Green's integral representations. In this case, Green's functions in the far-field are oseenlets, and Green's functions in the near-field are eulerlets. The outcome of this model is a wake in the Euler flow, which we call the Euler wake and which models momentum deficits giving both drag and lift. In particular, we apply the theory to uniform flow past a bluff circular cylinder at the high Reynolds number for mean-steady flow giving an Euler wake and drag on the body. There have been several recent studies investigating rotary oscillations of cylinders ${ }^{13}$ and their stable modes, ${ }^{14}$ as well as the vortex motion above a plane ${ }^{15}$ and an experimental PIV (Particle Image Velocimetry) investigation with dual step cylinders. ${ }^{16}$ Similarly, the focus of the application in this paper is circular cylinders with the emphasis on the mean-steady flow to use as a benchmark test.

The present study is structured as follows. In Sec. II, a statement of the problem is given where the Navier-Stokes equation is approximated in the far-field to produce the Oseen equation and in the near-field to produce the Euler equation. The relative size of the terms in the Navier-Stokes equation is carefully documented together with the size of the near- and far-fields in terms of the Reynolds number. The approach adopted is similar to that used by Sobey in Boundary Layer Theory (BLT) ${ }^{17}$ because both stretch the coordinate in the flow direction relative to the others. In Sec. III, the far-field Oseen integral representation in terms of oseenlets is given, and the high Reynolds number limit is taken. This is obtained by considering momentum losses in the wake that must be conserved and results in a constant distribution of Dirac delta functions along an infinite half-line in the wake. In Sec. IV, the near-field representation is given in terms of the eulerlets, and these are described and shown to be Green's functions of the Euler equation as well as matching to the far-field collapsed oseenlets. In Sec. V, the near and far-field integral representations are matched which results in the Euler flow representation of the velocity as an integral distribution of eulerlets over the body surface with strength given by the force distribution. In Sec. VI, the resulting Euler flow representations for attached flow around thin aerofoils, thin wings, and slender bodies are considered. These reduce to and so agree with standard aerodynamics potential flow models. In Sec. VII, separated flow past a circular cylinder is considered for the high Reynolds number, subcritical laminar flow at a Reynolds number of $1.1 \times 10^{4}$, and also turbulent flow at a Reynolds number of $8.4 \times 10^{6}$. It is shown that all the essential flow physics is captured. The streamlines compare well with flow visualization, and the pressure distribution around the circular cylinder is a reasonable match given the approximation made.

\section{STATEMENT OF THE PROBLEM}

We consider a steady, incompressible fluid of density $\rho$ and viscosity $\mu$ such that the Navier-Stokes equation

$$
\rho u_{j}^{\dagger} \frac{\partial u_{i}^{\dagger}}{\partial x_{j}^{\dagger}}=-\frac{\partial p^{\dagger}}{\partial x_{i}^{\dagger}}+\mu \frac{\partial^{2} u_{i}^{\dagger}}{\partial x_{j}^{\dagger} \partial x_{j}^{\dagger}}
$$

and continuity equation

$$
\frac{\partial u_{i}^{\dagger}}{\partial x_{i}^{\dagger}}=0
$$

hold throughout the fluid, where the symbol $\dagger$ means the dimensional and unperturbed values. So $u_{i}^{\dagger}$ and $p^{\dagger}$ are the fluid velocity and pressure, respectively, in Cartesian coordinates $x_{i}^{\dagger}, 1 \leq i \leq 2$ for two-dimensional flow and $1 \leq i \leq 3$ for three-dimensional flow. The Einstein convention of repeated suffix implying a summation is used such that $a_{i} b_{i}=a_{1} b_{1}+a_{2} b_{2}+a_{3} b_{3}$ in three-dimensions, for example.

Consider an exterior problem such that the far-field boundary condition is that the fluid velocity tends toward a uniform stream aligned to the $x_{1}^{\dagger}$ axis of magnitude $U$, and so

$$
u_{i}^{\dagger}=U \delta_{i 1},
$$

where $\delta_{i j}$ is the Kronecker delta. The perturbed velocity $u_{i}$ and perturbed pressure $p$ to the uniform stream satisfy $u_{i}^{\dagger}=U \delta_{i 1}+u_{i}, p^{\dagger}$ $=-(1 / 2) \rho U^{2}+p$, respectively, and so the perturbed Navier-Stokes equation is given by

$$
\rho U \frac{\partial u_{i}}{\partial x_{1}^{\dagger}}+\rho u_{j} \frac{\partial u_{i}}{\partial x_{j}^{\dagger}}=-\frac{\partial p}{\partial x_{i}^{\dagger}}+\mu \frac{\partial^{2} u_{i}}{\partial x_{j}^{\dagger} \partial x_{j}^{\dagger}} .
$$

In the far-field, the Navier-Stokes equation is approximated by the Oseen equation. The velocity $u_{i}$ and pressure $p$ then approximate to the Oseen velocity $u_{i}^{O}$ and Oseen pressure $p^{O}$, and the far-field boundary condition becomes

$$
u_{i}^{O} \rightarrow 0 \text { as }\left|x_{j}\right| \rightarrow \infty .
$$

Consider a near-field within which there is a fixed closed body. For the large Reynolds number, in the near-field, we approximate the Navier-Stokes equation again, and this time, we take the Euler equation to hold. The velocity $u_{i}$ and pressure $p$ then are approximated to the Euler velocity $u_{i}^{E}$ and the Euler pressure $p^{E}$. Therefore, the slip (impermeability) boundary condition for the Euler flow is considered such that

$$
u_{i}^{E} n_{i}=-U n_{1}
$$

for a normal vector $n_{i}$ to the body boundary $\partial \Sigma_{0}$. This assumes a negligibly thin viscous boundary layer just outside of which the Euler flow slips past the body. So, we take the Euler flow to be a leading order approximation to the viscous Navier-Stokes flow in the near field. This also means that we have not omitted the no-slip boundary condition; this still holds on the body, but instead, we apply the slip boundary condition at the edge of the boundary layer and then in the limit of a high Reynolds number assume the boundary layer thickness is vanishingly small. In future work, we shall consider including the boundary layer in the model and then match it to the near-field Euler flow outside the boundary layer. However, we argue that this is 
a secondary effect; for uniform flow past a circular cylinder, we shall find in Sec. VII that the drag originates from an Euler wake that produces a momentum deficit. Then, in the far-field, the wake diffuses, becoming the Oseen wake: Physically, in the near-field, diffusion is suppressed because the viscous diffusion term in the Navier-Stokes equations is negligible giving the Euler equations, which model the Euler wake. Then, in the far-field, the viscous diffusion term is no longer negligible giving the Oseen equations which model the Oseen wake. On the matching boundary, the Euler wake and Oseen wake match.

Finally, let us assume that the flow representation in the near and far-fields matches on a common shared matching boundary $\partial \Sigma_{M}$ for the large Reynolds number, so

$$
u_{i}^{O}=u_{i}^{E}, \text { on } \partial \Sigma_{M} .
$$

Before continuing, we need to demonstrate that the far-field does indeed approximate to the Oseen flow and the near-field to the Euler flow in the high Reynolds number limit, as seen in Sec. II A.

\section{A. Far-field}

Let the far-field Cartesian coordinates scale as $x_{1}^{\dagger}=(R e) l x_{1}^{O}$, $x_{\alpha}^{\dagger}=l x_{\alpha}^{O}$, see Fig. 4, where $l$ is a typical length dimension of the body, $R e=\rho U l / \mu$ is the Reynolds number which tends to infinity, and we denote the Greek letter suffixes starting from index 2. So, for a twodimensional flow, we have that $\alpha=2$, and for a three-dimensional flow, we have that $2 \leq \alpha \leq 3$. In the far-field, the flow is a small perturbation $\varepsilon$ to the uniform flow field such that $u_{i}=\varepsilon U u_{i}^{O}$ and $p=\varepsilon \rho U^{2} p^{O}$, where $p^{O}=p^{I}+(1 / R e) p^{I I}$. Substituting this into (4) and multiplying through by $\frac{(R e) l}{\varepsilon \rho U^{2}}$ give

$$
\begin{aligned}
\frac{\partial u_{i}^{O}}{\partial x_{1}^{O}}+ & \varepsilon u_{1}^{O} \frac{\partial u_{i}^{O}}{\partial x_{1}^{O}}+\varepsilon(R e) u_{\alpha}^{O} \frac{\partial u_{i}^{O}}{\partial x_{\alpha}} \\
= & -\frac{\partial p^{I}}{\partial x_{1}^{0}} \delta_{i 1}-\operatorname{Re} \frac{\partial p^{I}}{\partial x_{\alpha}^{O}} \delta_{i \alpha}-\frac{1}{R e} \frac{\partial p^{I I}}{\partial x_{1}^{O}} \delta_{i 1} \\
& -\frac{\partial p^{I I}}{\partial x_{\alpha}^{0}} \delta_{i \alpha}+\frac{1}{(R e)^{2}} \frac{\partial^{2} u_{i}^{O}}{\partial x_{1}^{O} \partial x_{1}^{O}}+\frac{\partial^{2} u_{i}^{O}}{\partial x_{\alpha}^{O} \partial x_{\alpha}^{O}} .
\end{aligned}
$$

The leading order term in large $R e$ is then $\operatorname{Re} \frac{\partial p^{I}}{\partial x_{\alpha}^{0}} \delta_{i \alpha}=0$, which means that $p^{I}\left(x_{1}\right)$, which is the same as the result found in BLT. ${ }^{17}$

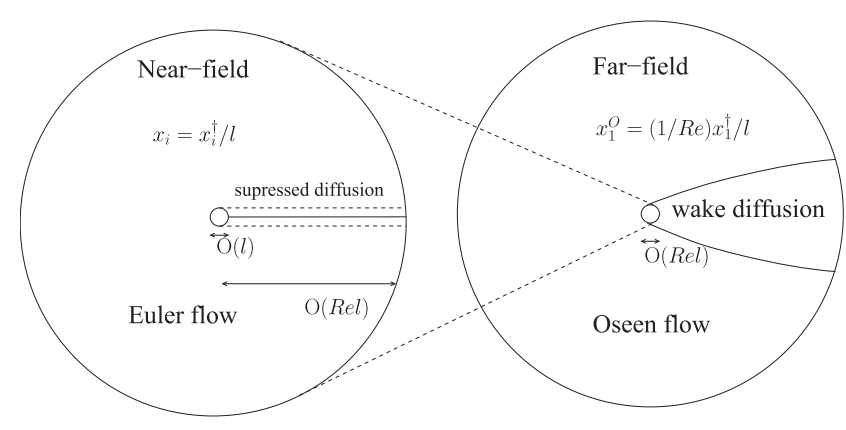

FIG. 4. The matching between the near and far fields
The next order gives

$$
\frac{\partial u_{i}^{O}}{\partial x_{1}^{O}}=-\frac{\partial p^{I}}{\partial x_{1}^{O}} \delta_{i 1}-\frac{\partial p^{I I}}{\partial x_{\alpha}^{O}} \delta_{i \alpha}+\frac{\partial^{2} u_{i}^{O}}{\partial x_{\alpha}^{O} \partial x_{\alpha}^{O}}
$$

since $\varepsilon u_{1}^{O} \frac{\partial u_{i}^{O}}{\partial x_{1}^{O}} \rightarrow 0$ and $\varepsilon(R e) u_{\alpha}^{O} \frac{\partial u_{i}^{O}}{\partial x_{\alpha}} \rightarrow 0$ when $R e \rightarrow \infty$ if we choose $\varepsilon$ small enough such that $\varepsilon=o(1 / R e)$, where little "o" means "of smaller order than." This means that all the terms in (9) are of the same order and none can be discounted. Rewriting in terms of dimensionless coordinates $x_{i}=x_{i}^{\dagger} / l$, then as $R e \rightarrow \infty$,

$$
\begin{aligned}
\frac{\partial^{2} u_{i}^{O}}{\partial x_{j} \partial x_{j}}= & \frac{\partial^{2} u_{i}^{O}}{\partial x_{\alpha}^{O} \partial x_{\alpha}^{O}}+\frac{1}{(\operatorname{Re})^{2}} \frac{\partial^{2} u_{i}^{O}}{\partial x_{1}^{O} \partial x_{1}^{O}}=\frac{\partial^{2} u_{i}^{O}}{\partial x_{\alpha}^{O} \partial x_{\alpha}^{O}}, \\
\operatorname{Re} \frac{\partial p^{O}}{\partial x_{i}} & =\frac{\partial p^{O}}{\partial x_{1}^{O}} \delta_{i 1}+\operatorname{Re} \frac{\partial p^{O}}{\partial x_{\alpha}^{O}} \delta_{i \alpha} \\
& =\frac{\partial p^{I}}{\partial x_{1}^{O}} \delta_{i 1}+\frac{1}{\operatorname{Re}} \frac{\partial p^{I I}}{\partial x_{1}^{O}} \delta_{i 1}+\frac{\partial p^{I I}}{\partial x_{\alpha}^{O}} \delta_{i \alpha} \\
& =\frac{\partial p^{I}}{\partial x_{1}^{O}} \delta_{i 1}+\frac{\partial p^{I I}}{\partial x_{\alpha}^{O}} \delta_{i \alpha},
\end{aligned}
$$

and

$$
R e \frac{\partial u_{i}^{O}}{\partial x_{1}}=\frac{\partial u_{i}^{O}}{\partial x_{1}^{O}} .
$$

Putting (10)-(12) into (9) and dividing through by Re give

$$
\frac{\partial u_{i}^{O}}{\partial x_{1}}=-\frac{\partial p^{O}}{\partial x_{i}}+\frac{1}{R e} \frac{\partial^{2} u_{i}^{O}}{\partial x_{j} \partial x_{j}} .
$$

Letting a comma denote a differentiation such that $f_{, i}=\frac{\partial f}{\partial x_{i}}$, this is the Oseen equation

$$
u_{i, 1}^{O}=-p_{, i}^{O}+(1 / R e) u_{i, j j}^{O}
$$

such that as $R e \rightarrow \infty$, all the terms are of the same order and none can be discounted from (9).

\section{B. Near-field}

Considering near-field dimensionless variables $x_{i}^{\dagger}=l x_{i}, u_{i}^{\dagger}=$ $U u_{i}, p=\rho U^{2} p$, then substituting into (4), and multiplying through by $l /\left(\rho U^{2}\right)$ give

$$
u_{i, 1}+u_{j} u_{i, j}=-p_{, i}+(1 / R e) u_{i, j j} .
$$

In the limit as $R e \rightarrow \infty$, this becomes the Euler equation

$$
u_{i, 1}^{E}+u_{j}^{E} u_{i, j}^{E}=-p_{, i}^{E} \text {. }
$$

The near-field and far-field then match on the common boundary $x_{1}^{\dagger}=(R e) l$; see Fig. 4 .

\section{FAR-FIELD OSEEN REPRESENTATION} satisfy ${ }^{2}$

Green's functions for the Oseen equation, called oseenlets,

$$
u_{i k, 1}^{O}=-p_{k, i}^{O}+(1 / R e) u_{i k, j j}^{O}-\delta \delta_{i k}, \quad u_{i k, i}^{O}=0,
$$


where $u_{i k}^{O}$ and $p_{k}^{O}$ are the velocity and pressure, respectively, of the $k$ th oseenlet and the $k$ th oseenlet velocity $u_{i k}^{O}$ has vector components directed along the $x_{i}$ axes. The function $\delta$ is the Dirac delta function such that the spatial integration across it is unity $\int_{\Sigma} \delta d \Sigma=1$.

Oseen gives the oseenlets in two-dimensional flow as

$$
\begin{aligned}
& u_{i 1}^{O}=\frac{1}{2 \pi}\left\{\left[\ln r+e^{k x_{1}} K_{0}(k r)\right]_{, i}-2 k e^{k x_{1}} K_{0}(k r) \delta_{i 1}\right\}, \\
& u_{i 2}^{O}=\frac{1}{2 \pi} \varepsilon_{i j 3}\left[\ln r+e^{k x_{1}} K_{0}(k r)\right]_{, j}, p_{k}^{O}=-\frac{1}{2 \pi}[\ln r]_{, k},
\end{aligned}
$$

where $\varepsilon_{i j k}$ is the Levi-Civita symbol such that $\varepsilon_{123}=\varepsilon_{231}=\varepsilon_{312}=1$, $\varepsilon_{132}=\varepsilon_{213}=\varepsilon_{321}=-1$, and $\varepsilon_{i j k}=0$ otherwise. In three-dimensional flow, Oseen gives the oseenlets as

$$
\begin{aligned}
& u_{i k}^{O}=\phi_{k, i}^{O}-\chi_{k, i}^{O}+2 k \chi_{1}^{O} \delta_{i k}, \quad p_{k}^{O}=-\frac{1}{4 \pi}[1 / R]_{, k}, \\
& \phi_{k}^{O}=\frac{1}{4 \pi}\left[\ln \left(R-x_{1}\right)\right]_{, k}, \quad \chi_{k}^{O}=\frac{1}{4 \pi} e^{-k\left(R-x_{1}\right)}\left[\ln \left(R-x_{1}\right)\right]_{, k} .
\end{aligned}
$$

We call the constant term $k$ (not the suffix index) the dimensionless wake number such that $k=(R e) / 2$ (the dimensional wake number is $k / l$ and so has dimension $1 / l$ for length $l$ ). (The counterpart is the wave number $k$ arising from a sign change in the differential equation terms leading to wave rather than wake solutions. In the same way that the wave number gives the expected wave size, the wake number gives the expected wake size.) The modified Bessel function of the first kind of order 0 is denoted by $K_{0}$. The two-dimensional radial length $r$ and the three-dimensional radial length $R$ are such that $x_{i} x_{i}=r^{2}$ in two-dimensions and $x_{i} x_{i}=R^{2}$ in three-dimensions.

Using (14) and (17), Oseen then gives the following Green's integral in the Oseen flow:

$$
\begin{aligned}
\int_{\Sigma^{O}}[ & \left\{u_{i ; 1}^{O}+p_{; i}^{O}-(1 / R e) u_{i ; j j}^{O}\right\} u_{i k}^{O} \\
& \left.-\left\{u_{i k, 1}^{O}+p_{k, i}^{O}-(1 / R e) u_{i k, j j}^{O}+\delta \delta_{i k}\right\} u_{i}^{O}\right] d \Sigma^{\prime}=0,
\end{aligned}
$$

where we denote the semicolon to represent a derivative with respect to the variable of integration (defined in more detail in this following paragraph) and $d \Sigma^{\prime}=d x_{1}^{\prime} d x_{2}^{\prime} d x_{3}^{\prime}$ (in three-dimensions, for example) refers to an element of the space $\Sigma^{O}$ over which the integration takes place; the integral is therefore parameterized by points denoted by $x_{i}^{\prime}$, the variable of integration. In this integral, the velocity and pressure are therefore functions of $x_{i}^{\prime}$, but the oseenlet Green's functions are given as functions of $\left(x_{i}-x_{i}^{\prime}\right)$. In the analysis, two types of differentiations are therefore used, a differentiation with respect to $x_{i}$ but also differentiation with respect to $x_{i}^{\prime}$, the coordinate position of the variable of integration. In the Einstein notation, we have used comma "," to denote a differentiation with respect to $x_{i}$, so we will use a semicolon ";" as shorthand to denote differentiation with respect to $x_{i}^{\prime}$. (This is different from their use in the Einstein tensor notation as representing the contravariant and covariant derivatives.) So, $u_{i k, j}^{O}=-u_{i k ; j}^{O}$, for example. Rearranging and evaluating the Dirac delta function term in (20), a representation for the far-field Oseen velocity is then given by

$$
\begin{aligned}
u_{k}^{O}= & \int_{\Sigma^{O}}\left[\left(u_{i}^{O} u_{i k}^{O}\right)_{; 1}+p_{; i}^{O} u_{i k}^{O}-p_{k, i}^{O} u_{i}^{O}\right. \\
& \left.+(1 / R e)\left(u_{i k ; j}^{O} u_{i}^{O}-u_{i ; j j}^{O} u_{i k}^{O}\right)\right] d \Sigma^{\prime} .
\end{aligned}
$$

We now express this integral in terms of the velocity potential $\phi$ rather than the pressure $p$; noting that from (18), the twodimensional oseenlets can be expressed in the form $u_{i k}^{O}=\phi_{k, i}^{O}+w_{i k}^{O}$ such that $\phi_{1}^{O}=\frac{1}{2 \pi} \ln r$ and $\phi_{2}^{O}=-\frac{1}{2 \pi} \theta$ for the polar angle $\theta$ in the polar coordinate representation $x_{1}=r \cos \theta, x_{2}=r \sin \theta$. From (19), the three-dimensional oseenlet velocities $u_{i k}^{O}$ are already expressed in the form of a velocity potential derivative $\phi_{k, i}^{O}$ added to a wake velocity $w_{i k}^{O}$, and for both two- and three-dimensions, the following expression for the oseenlet pressure holds:

$$
p_{k}^{O}=-\phi_{k, 1}^{O}
$$

Due to the linearity of (21), it follows that the same HelmholtzHodge vector decomposition (fundamental theorem of vector calculus) also holds for the Oseen velocity such that

$$
u_{i}^{O}=\phi_{, i}^{O}+w_{i}^{O} .
$$

In this context, the wake velocity is further decomposed by a wake potential $\chi^{0}$ and this is called the Lamb-Goldstein velocity decomposition which when substituted into the Oseen equation (14) gives the relation for pressure

$$
p^{O}=-\phi_{, 1}^{O}
$$

However, we note that in general, this vector decomposition is undefined in a shadow wake region. ${ }^{1}$ In two-dimensional flow, we can make the potential term $\theta$ single-valued instead of multivalued by introducing a branch cut along the positive $x_{1}$ axis across which the potential is discontinuous. In this way, the potential is then defined in the shadow region for two-dimensional flow. The physical interpretation of this is a circulation related to lift. However, in threedimensional flow, the whole shadow region may have an undefined potential. ${ }^{1}$ The physical interpretation of this region is a vortex wake related to lift. However, we also see that by pairing the potential velocity and wake velocity together, the resultant velocity $u_{i}^{O}$ is now defined in the shadow region. ${ }^{1}$ Substituting (22) and (24) into (21) and taking the limit as $R e \rightarrow \infty$, we get

$$
u_{k}^{O}=\int_{\Sigma^{O}}\left[\left(u_{i}^{O} u_{i k}^{O}\right)_{; 1}-\phi_{; 1 i}^{O} u_{i k}^{O}+\phi_{k, 1 i}^{O} u_{i}^{O}\right] d \Sigma^{\prime} .
$$

In the far-field wake, we have $x_{1}=O(R e), x_{2}=O(1)$, and so $x_{2} / x_{1}=$ $O(1 / R e) \rightarrow 0$ as $R e \rightarrow \infty$, and so the wake oseenlets $w_{i k}^{O}=u_{i k}^{O}-\phi_{k, i}^{O}$ collapse onto a wake line and simplify. We determine this form by looking at wake integrals next.

\section{A. Wake integrals}

The potential flow of the oseenlet generates a force, and the viscous flow of the oseenlet also generates a force, and from (17), in total, these generate unit force in the $k$-direction. The contributions from each have been determined, in particular, for the three-dimensional lift, ${ }^{7,9}$ and are listed in Table I.

For the wake velocity, the contributions can be calculated from far-field wake momentum deficits, ${ }^{18}$ which are proportional to integrations of the velocity in the cross-sectional plane normal to the $x_{1}$-direction. These integral contributions must still hold in the high Reynolds number limit so that the oseenlet (17) also still holds. 
TABLE I. The force contributions to the oseenlets.

\begin{tabular}{lcc}
\hline \hline & Velocity potential & Wake velocity \\
\hline 2-D drag & 0 & 1 \\
2-D lift & 1 & 0 \\
3-D drag & 0 & 1 \\
3-D lift/side force & $1 / 2$ & $1 / 2$ \\
\hline \hline
\end{tabular}

\section{Two-dimensions}

In the far-field, the $x_{1}$ scaling is of order $O(R e)$, whereas the $x_{\alpha}$ scaling is of order $O(1)$. So, for large $R e=2 k$, we approximate for $x_{1}>>\left|x_{\alpha}\right|$. This gives from the work of Abramowitz and Stegun ${ }^{19}$

$$
\begin{aligned}
e^{k x_{1}} K_{0}(k r) & \sim \sqrt{\frac{\pi}{2 k r}} e^{-k\left(r-x_{1}\right)} \sim \sqrt{\frac{\pi}{2 k r}} e^{-k x_{2}^{2} / 2 x_{1}} \\
& =\sqrt{\frac{\pi}{2 k r}} e^{-\eta^{2}}, \quad \eta=\sqrt{\frac{k}{2 x_{1}}} x_{2},
\end{aligned}
$$

where $\eta$ is the same as the boundary layer variable, except in BLT $x_{1}^{B L T}=O(1), x_{2}^{B L T}=O\left(\frac{1}{\sqrt{R e}}\right),{ }^{17}$ whereas in the far wake $x_{1}=O(R e)$, $x_{2}=O(1)$. However, both give the same parabolic wake/boundary layer profile described by $\eta$ due to stretching of the $x_{1}$ coordinate relative to the others. In the near-field, the scaling is $x_{i}=O(1)$, and so $\eta=O(\sqrt{R e})$. For large $R e, \eta$ is large and so diffusion is suppressed, meaning that the wake does not diffuse.

The two-dimensional drag is given as a far-field momentum deficit; see, for example, Ref. 18. For the two-dimensional oseenlet, from Table I, unit drag comes from the wake velocity and so

$$
-\int_{-\infty}^{\infty} w_{11}^{O} d x_{2} \sim \frac{1}{\sqrt{\pi}} \int_{-\infty}^{\infty} e^{-\eta^{2}} d \eta=1=\int_{-\infty}^{\infty} H\left(x_{1}\right) \delta\left(x_{2}\right) d x_{2},
$$

where $H\left(x_{1}\right)$ is the Heaviside function $H\left(x_{1}\right)=0$ for $x_{1}<0$ and $H\left(x_{1}\right)$ $=1$ for $x_{1} \geq 0$. So, the two-dimensional oseenlet collapses to

$$
\begin{aligned}
& u_{i 1}^{O}=\frac{1}{2 \pi}[\ln r]_{, i}-H\left(x_{1}\right) \delta\left(x_{2}\right) \delta_{i 1}, \\
& u_{i 2}^{O}=\frac{1}{2 \pi} \varepsilon_{i j 3}[\ln r]_{, j}=-\frac{1}{2 \pi} \theta_{, i}, \\
& p_{k}^{O}=\frac{1}{2 \pi}[\ln r]_{, k} .
\end{aligned}
$$

\section{Three-dimensions}

For $x_{1}>\left|x_{\alpha}\right|$, then $k\left(R-x_{1}\right)$ approximates to $k\left(R-x_{1}\right) \sim$ $k r^{* 2} / 2 x_{1}=\eta^{* 2}, \eta^{*}=\sqrt{\frac{k}{2 x_{1}}} r^{*}, r^{*}=\sqrt{x_{2}^{2}+x_{3}^{2}}$. So in the far-field wake, the oseenlet potentials become

$$
\phi_{k}^{O} \sim \frac{1}{4 \pi}\left[2 \ln r^{*}-\ln 2 x_{1}\right]_{, k} \quad \chi_{k}^{O} \sim \frac{1}{4 \pi} e^{-\eta^{* 2}}\left[2 \ln r^{*}-\ln 2 x_{1}\right]_{, k} .
$$

Drag: To calculate the drag, we need the limiting wake velocity $w_{11}^{O}=-\chi_{1,1}^{O}+2 k \chi_{1}^{O}$ from (19), where $\chi_{1}^{O} \sim-\frac{1}{4 \pi x_{1}} e^{-\eta^{* 2}}$, and so $\chi_{1,1}^{O} \sim-\frac{1}{4 \pi x_{1}} e^{-\eta^{* 2}}\left[-2 \eta^{*} \eta_{, 1}^{*}-\frac{1}{x_{1}}\right]$. Looking at the order of the terms, $\eta_{, 1}^{*}=\sqrt{\frac{k}{2 x_{1}}} r^{*}\left(-\frac{1}{2 x_{1}}\right)=O(1 / R e)$ and $\frac{1}{x_{1}}=O\left(\frac{1}{R e}\right)$, the unit drag of the oseenlet is

$$
\begin{aligned}
-\int_{0}^{2 \pi} & \int_{0}^{\infty} w_{11}^{O} r^{*} d r^{*} d \theta^{*} \\
& =-\int_{0}^{2 \pi} \int_{0}^{\infty} 2 k \chi_{1}^{O} r^{*} d r^{*} d \theta^{*}\left(1+O\left(\frac{1}{(R e)^{2}}\right)\right) \\
& \sim \frac{k}{x_{1}} \int_{0}^{\infty} e^{-\eta^{* 2}} r^{*} d r^{*} \\
& =1=\int_{-\infty}^{\infty} \int_{-\infty}^{\infty} H\left(x_{1}\right) \delta\left(x_{2}\right) \delta\left(x_{3}\right) d x_{2} d x_{3},
\end{aligned}
$$

where the polar angle $\theta^{*}$ is defined as $x_{2}=r^{*} \cos \theta^{*}$ and $x_{3}=r^{*} \sin \theta^{*}$. So the three-dimensional drag oseenlet becomes

$$
u_{i 1}^{O}=-\frac{1}{4 \pi}\left[\frac{1}{R}\right]_{, i}-H\left(x_{1}\right) \delta\left(x_{2}\right) \delta\left(x_{3}\right) \delta_{i 1} .
$$

Lift: From Ref. 7, half the lift contribution comes from the wake flow, as presented in Table I. This is confirmed by the far-field wake evaluation next. For the evaluation, we require $\chi_{2,2}^{O}$ in the far-field wake. We have

$$
\chi_{2}^{O} \sim \frac{1}{4 \pi} e^{-\eta^{* 2}} \frac{2 x_{2}}{r^{* 2}}
$$

and so

$$
\chi_{2,2}^{O} \sim \frac{1}{2 \pi}\left\{e^{-\eta^{* 2}}\left(\frac{1}{r^{* 2}}-\frac{2 \cos ^{2} \theta^{*}}{r^{* 2}}-\frac{k}{x_{1}} \cos ^{2} \theta^{*}\right)\right\} .
$$

Substituting this into the momentum deficit integration for lift

$$
-\int_{\partial \Sigma^{O}} w_{22}^{O} d \sigma=\int_{0}^{2 \pi} \int_{0}^{\infty}\left(\chi_{2,2}^{O}-2 k \chi_{1}^{O}\right) r^{*} d r^{*} d \theta^{*}
$$

where $\partial \Sigma^{O}$ is an integration across the whole of the 2-dimensional plane located at constant $x_{1}$, gives a contribution from the first term in the integrand of

$$
\begin{aligned}
\int_{0}^{\infty} & \int_{0}^{2 \pi} \chi_{2,2}^{O} r^{*} d \theta^{*} d r^{*} \\
& =\frac{1}{2 \pi} \int_{0}^{\infty} \int_{0}^{2 \pi} e^{-\eta^{* 2}}\left(\frac{1}{r^{* 2}}-\frac{2 \cos ^{2} \theta^{*}}{r^{* 2}}-\frac{k}{x_{1}} \cos ^{2} \theta^{*}\right) r^{*} d \theta^{*} d r^{*} \\
& =-\int_{0}^{\infty} \eta^{*} e^{-\eta^{* 2}} d \eta^{*}=-1 / 2 .
\end{aligned}
$$

The contribution from the second term in the integrand is the same as for the drag calculation, and so the total contribution gives

$$
\begin{aligned}
-\int_{\partial \Sigma^{O}} w_{22}^{O} d \sigma & =-1 / 2+1=1 / 2 \\
& =1 / 2 \int_{-\infty}^{\infty} \int_{-\infty}^{\infty} H\left(x_{1}\right) \delta\left(x_{2}\right) \delta\left(x_{3}\right) d x_{2} d x_{3} .
\end{aligned}
$$

So the three-dimensional lift oseenlet becomes

$$
u_{i \alpha}^{O}=\frac{1}{4 \pi}\left[\ln \left(R-x_{1}\right)\right]_{, \alpha i}-(1 / 2) H\left(x_{1}\right) \delta\left(x_{2}\right) \delta\left(x_{3}\right) \delta_{i \alpha} .
$$

\section{NEAR-FIELD EULER REPRESENTATION}

The eulerlets are defined as the unit force fundamental solutions such that

$$
u_{i k, 1}^{E}+\left[u_{j}^{E} u_{i, j}^{E}\right]_{k}=-p_{k, i}^{E}-\delta \delta_{i k}
$$


where $\left[u_{j}^{E} u_{i, j}^{E}\right]_{k}$ is the $k$ th eulerlet expression for the term $u_{j}^{E} u_{i, j}^{E}$, and $\delta=\delta\left(x_{1}\right) \delta\left(x_{2}\right)$ in two-dimensions and $\delta=\delta\left(x_{1}\right) \delta\left(x_{2}\right) \delta\left(x_{3}\right)$ in three-dimensions. We shall show that (38) is satisfied by the two-dimensional eulerlets given by

$$
u_{i 1}^{E}=\frac{1}{2 \pi}[\ln r]_{, i}-H\left(x_{1}\right) \delta\left(x_{2}\right) \delta_{i 1}, \quad u_{i 2}^{E}=-\frac{1}{2 \pi}[\theta]_{, i}
$$

and the three-dimensional eulerlet velocity

$$
\begin{aligned}
& u_{i 1}^{E}=-\frac{1}{4 \pi}\left[\frac{1}{R}\right]_{, i}-H\left(x_{1}\right) \delta\left(x_{2}\right) \delta\left(x_{3}\right) \delta_{i 1}, \\
& u_{i \alpha}^{E}=\frac{1}{4 \pi}\left[\ln \left(R-x_{1}\right)\right]_{, \alpha i}-(1 / 2) H\left(x_{1}\right) \delta\left(x_{2}\right) \delta\left(x_{3}\right) \delta_{i \alpha} .
\end{aligned}
$$

From (28), (31), and (37), it is clear that these eulerlets match to the respective oseenlets on the matched boundary such that $u_{i k}^{E}=u_{i k}^{O}$ there. The streamlines for the eulerlets are represented pictorially in Figs. (1), 2(a), and 2(b) centered at the origin. For the threedimensional lift eulerlet described in Fig. 2(b), the $x_{1}$-axis represents a collapsed circulatory flow.

In Sec. IV A, we shall show that (39) and (40) satisfy the continuity equation and then calculate the eulerlet vorticity and forces.

\section{A. Continuity equation and vanishing divergence}

In two-dimensional flow, the drag eulerlet gives divergence

$$
u_{i 1, i}^{E}=\frac{1}{2 \pi}[\ln r]_{, i i}-\delta=0
$$

since $H^{\prime}\left(x_{1}\right)=\delta\left(x_{1}\right)$ and $\delta=\delta\left(x_{1}\right) \delta\left(x_{2}\right)$. From symmetry, the continuity and divergence for the lift eulerlet are identically zero. [The resulting domain integration, about a sphere in three-dimensions and a circle in two-dimensions, centered at the origin transforms to boundary integrals over the sphere surface/circle circumference upon application of the divergence theorem. However, all the resulting integrands are antisymmetric (odd), and so the domain integration is identically zero.] So

$$
u_{i 2, i}^{E}=0 .
$$

Similarly, in three-dimensions, the drag eulerlet gives divergence

$$
u_{i 1, i}^{E}=\left[-\frac{1}{4 \pi R}\right]_{, i i}-\delta=0,
$$

and again from symmetry arguments, the lift and side force eulerlet gives zero divergence

$$
u_{i \alpha, i}^{E}=0
$$

where $\alpha=2,3$.

\section{B. Eulerlet vorticity}

Again, by invoking symmetry, the vorticity for the drag eulerlet for both two- and three-dimensional flows is identically zero, so

$$
\varepsilon_{i j k} u_{k 1, j}^{E}=0 .
$$

However, the vorticity for the lift eulerlet in two-dimensions gives

$$
\begin{aligned}
\varepsilon_{3 j k} u_{k 2, j}^{E} & =\varepsilon_{3 j k} \varepsilon_{k q 3} \frac{1}{2 \pi}[\ln r]_{j q} \\
& =\frac{1}{2 \pi}\left(\delta_{3 q} \delta_{j 3}-\delta_{33} \delta_{j q}\right)[\ln r]_{j q}=-\frac{1}{2 \pi}[\ln r]_{, j j}=-\delta .
\end{aligned}
$$

Similarly, by invoking symmetry in three-dimensions, the vorticity for the 2-eulerlet is

$$
\begin{aligned}
\varepsilon_{i j k} u_{k 2, j}^{E} & =\varepsilon_{i j k}\left(\phi^{*}, 2 k j\right. \\
& =w_{i 3}^{E}\left\{\left(\phi^{*}, 221-\phi^{*}, 212\right)=\varepsilon_{3 j k} \delta_{i 3}\left(\phi^{*}, 2 k j+w_{k 2, j}^{E}\right)\right. \\
& =\delta / 2\}
\end{aligned}
$$

where we have let $\phi^{*}=\frac{1}{4 \pi} \ln \left(R-x_{1}\right)$. However, we note that

$$
\begin{aligned}
& \int_{\Sigma}\left(\phi_{, 221}^{*}-\phi_{, 212}^{*}\right) d \Sigma=\int_{\partial \Sigma}\left(\phi_{, 22}^{*} n_{1}-\phi_{, 21}^{*} n_{2}\right) d \sigma \\
& =\int_{\partial \Sigma}\left(\left((1 / 2) \phi_{, 22}^{*}+(1 / 2) \phi_{, 33}^{*}\right) n_{1}-\phi_{, 21}^{*} n_{2}\right) d \sigma \\
& =\int_{\partial \Sigma}\left(\left((-1 / 2) \phi,{ }_{11}^{*}\right) n_{1}-\phi,{ }_{12}^{*} n_{2}\right) d \sigma \\
& =\int_{\partial \Sigma}\left(\left((-1 / 2)(1 / 3) \phi,{ }_{1 j}^{*}\right) n_{j}-(1 / 3) \phi,{ }_{1 j}^{*} n_{j}\right) d \sigma \\
& =-1 / 2 \int_{\partial \Sigma} \phi,{ }_{1 j}^{*} n_{j} d \sigma \\
& =-1 / 2 \int_{\partial \Sigma}[-1 / 4 \pi R]_{, j} n_{j} d \sigma \\
& =-1 / 2 \int_{\Sigma}[-1 / 4 \pi R]_{, j j} d \Sigma=-1 / 2 \int_{\Sigma} \delta d \Sigma,
\end{aligned}
$$

where $\partial \Sigma$ is the boundary of $\Sigma$. (It is also noted that when performing these operations, the derivatives over the space $\Sigma$ that include the eulerlet origin are not commutative, whereas over the boundary of the space $\partial \Sigma$ that encloses the eulerlet they are.) Using this result in (47) gives

$$
\varepsilon_{i j k} u_{k 2, j}^{E}=\delta_{i 3}(-\delta / 2-\delta / 2)=-\delta \delta_{i 3} .
$$

Similarly, the vorticity for the 3 -eulerlet is

$$
\begin{aligned}
\varepsilon_{i j k} u_{k 3, j}^{E} & =\varepsilon_{i j k}\left(\phi^{*}{ }_{3 k j}+w_{k 3, j}^{E}\right)=\delta_{i 2}\left\{\left(\phi^{*}, 313-\phi^{*}, 331\right)-w_{33,1}^{E}\right\} \\
& =\delta_{i 2}(\delta / 2+\delta / 2)=\delta \delta_{i 2} .
\end{aligned}
$$

Putting all these results together then gives the vorticity expression

$$
\begin{aligned}
u_{i k, j}^{E}-u_{j k, i}^{E} & =0 \text { for } k=1 \\
& =-\delta \delta_{i k} \delta_{j 1}+\delta \delta_{j k} \delta_{i 1} \text { for } k \neq 1
\end{aligned}
$$

\section{Eulerlet force distribution}

Define the eulerlet force distribution as $f_{i k}=\phi_{k, 1 i}^{E}-u_{i k, 1}^{E}$. To find the eulerlet pressure and force, we first evaluate this relation.

For $k=1, w_{1 k, i}^{E}=-\delta \delta_{i 1}=-\delta \delta_{i k}$ from (39) and (40), and so substituting this into (51) with $j=1$ gives

$$
\begin{aligned}
& u_{i k, 1}^{E}-u_{1 k, i}^{E}=0, \phi_{k, 1 i}^{E}+w_{1 k, i}^{E}-u_{i k, 1}^{E}=0, \\
& \phi_{k, 1 i}^{E}-u_{i k, 1}^{E}=-w_{1 k, i}^{E}=\delta \delta_{i k} .
\end{aligned}
$$


For $k \neq 1, w_{1 k}^{E}=0$ and so $w_{1 k, i}^{E}=0$ from (39) and (40), and so substituting this into (51) with $j=1$ gives

$$
\begin{aligned}
& u_{i k, 1}^{E}-u_{1 k, i}^{E}=-\delta \delta_{i k}, \quad \phi_{k, 1 i}^{E}+w_{1 k, i}^{E}-u_{i k, 1}^{E}=\delta \delta_{i k}, \\
& \phi_{k, 1 i}^{E}-u_{i k, 1}^{E}=\delta \delta_{i k} .
\end{aligned}
$$

So for all $k$, we have the relation

$$
f_{i k}=\phi_{k, 1 i}^{E}-u_{i k, 1}^{E}=\phi_{k, 1 i}^{E}-\phi_{k, 11}^{E}-w_{i k, 1}^{E}=\delta \delta_{i k} .
$$

The potential part gives a vorticity expression related to circulation, and the wake part gives a velocity deficit in the wake related to momentum loss.

\section{Eulerlet pressure}

Substituting into the eulerlet equation (38) gives $\phi_{k, 1 i}^{E}-\delta \delta_{i k}+$ $\left[u_{j}^{E} u_{i, j}^{E}\right]_{k}=-p_{k, i}^{E}-\delta \delta_{i k}$, and so the eulerlet pressure satisfies

$$
p_{k, i}^{E}+\left[u_{j}^{E} u_{i, j}^{E}\right]_{k}=-\phi_{k, 1 i}^{E} .
$$

For $\mathbf{x} \neq 0$ (where $\mathbf{x}=\left(x_{1}, x_{2}\right)$ in two-dimensions and $\left(x_{1}, x_{2}, x_{3}\right)$ in three-dimensions), we have $u_{i k, j}^{E}=u_{j k, i}^{E}$ from (51). Substituting into (55) and integrating up give

$$
p_{k}^{E}+(1 / 2)\left[u_{j}^{E} u_{j}^{E}\right]_{k}+\phi_{k, 1}^{E}=0,
$$

which is the Bernoulli equation.

\section{E. Eulerlet force}

The eulerlet force is

$$
\begin{aligned}
F_{i k} & =\int_{\Sigma}\left(-p_{k, i}^{E}-u_{i k, 1}^{E}-\left[u_{j}^{E} u_{i, j}^{E}\right)\right]_{k} d \Sigma=\int_{\Sigma}\left(\phi_{k, 1 i}^{E}-u_{i k, 1}^{E}\right) d \Sigma \\
& =\int_{\Sigma} f_{i k} d \Sigma=\int_{\Sigma} \delta \delta_{i k} d \Sigma=\delta_{i k} .
\end{aligned}
$$

So the $k$-eulerlet gives a unit force in the $k$-direction.

\section{F. Green's integral representation by eulerlets}

From the Euler equation (16) and the eulerlet equation (38), applying Green's integral construction that Oseen used but for the Euler flow, we get

$\int_{\Sigma^{E}}\left[\left\{u_{i ; 1}^{E}+p_{; i}^{E}+u_{j}^{E} u_{i ; j}^{E}\right\} u_{i k}^{E}-\left\{u_{i k, 1}^{E}+p_{k, i}^{E}+\left[u_{j}^{E} u_{i, j}^{E}\right]_{k}+\delta \delta_{i k}\right\} u_{i}^{E}\right] d \Sigma^{\prime}=0$

for some space $\Sigma^{E}$ in the Euler flow over which the integration takes place. [It is noted that the Dirac delta function in (58) assumes that (58) can be changed to a boundary integral and the Dirac delta function then represents the contribution around the point $\mathbf{x}$. This is only demonstrated once it is matched.] Rearranging (58) then gives a representation for the near-field Euler velocity as

$$
u_{k}^{E}=\int_{\Sigma^{E}}\left[\left(u_{i}^{E} u_{i k}^{E}\right)_{; 1}+\left(p_{; i}^{E}+u_{j}^{E} u_{i ; j}^{E}\right) u_{i k}^{E}-\left(p_{k, i}^{E}+\left[u_{j}^{E} u_{i, j}^{E}\right]_{k}\right) u_{i}^{E}\right] d \Sigma^{\prime} .
$$

From (55), we have that $p_{k}^{E}=-\phi_{k, 1}^{E}-\left[u_{j}^{E} u_{i, j}^{E}\right]_{k}$ and from (16) that $u_{i, 1}^{E}+u_{j}^{E} u_{i, j}^{E}=-p_{, i}^{E}$, so (59) becomes

$$
u_{k}^{E}=\int_{\Sigma^{E}}\left[\left(u_{i}^{E} u_{i k}^{E}\right)_{; 1}-u_{i ; 1}^{E} u_{i k}^{E}+\phi_{k, 1 i}^{E} u_{i}^{E}\right] d \Sigma^{\prime} .
$$

\section{G. Matching of the near and far-fields}

We require the velocity representations (25) and (60) to match. So, we let $u_{i, 1}^{E}=\phi_{, 1 i}^{E}$ in the matching region. In the matching region, $u_{i}^{E}=\phi_{, i}^{E}+w_{i}^{E}$ since $u_{i}^{E}=u_{i}^{O}$. This means that the matching also additionally requires $w_{i, 1}^{E}=0$. So the Euler wake has an unchanging cross-sectional profile in the plane of constant $x_{1}$. This then gives the Euler velocity representation in the matching region the same as the Oseen velocity representation (25) to be

$$
u_{k}^{E}=\int_{\Sigma^{E}}\left[\left(u_{i}^{E} u_{i k}^{E}\right)_{; 1}-\phi_{; 1 i}^{E} u_{i k}^{E}+\phi_{k, 1 i}^{E} u_{i}^{E}\right] d \Sigma^{\prime} .
$$

It is noted that a consequence of the matching is that (61) is linear rather than nonlinear. Furthermore, since the eulerlets are solutions to the Euler equation, then, we can use (61) to continue the Euler velocity $u_{k}^{E}$ into the near-field, and by doing so, (61) now represents the near-field Euler velocity $u_{k}^{E}$ everywhere in the near field.

\section{EULER FLOW EXPRESSIONS}

Using this formulation, we can now work out expressions for the Euler velocity by an integral distribution of eulerlets whose strength is the force distribution over the body.

\section{A. Euler velocity}

Applying the divergence theorem to (61) gives

$$
\begin{aligned}
u_{k}^{E} & =\int_{\partial \Sigma}\left[u_{i}^{E} u_{i k}^{E} n_{1}^{\Sigma}-\phi_{; 1}^{E} u_{i k}^{E} n_{i}^{\Sigma}+\phi_{k ; 1}^{E} u_{i}^{E} n_{i}^{\Sigma}\right] d \sigma^{\prime} \\
& =\int_{\partial \Sigma}\left[-u_{i}^{E} u_{i k}^{E} n_{1}+\phi_{; 1}^{E} u_{i k}^{E} n_{i}+\phi_{k, 1}^{E} u_{i}^{E} n_{i}\right] d \sigma^{\prime} \\
& =\int_{\partial \Sigma}\left[-u_{i}^{E} u_{k i}^{E} n_{1}+\phi_{; 1}^{E} u_{k i}^{E} n_{i}+\phi_{1, k}^{E} u_{i}^{E} n_{i}\right] d \sigma^{\prime} \\
& =\int_{\partial \Sigma}\left[f_{i} u_{k i}^{E}+u_{i}^{E} n_{i} \phi_{1, k}^{E}\right] d \sigma^{\prime},
\end{aligned}
$$

where $n_{i}^{\Sigma}$ is the outward pointing normal to the space $\Sigma$ and $n_{i}=$ $-n_{i}^{\Sigma}$ is the outward pointing normal to the surface $\partial \Sigma$; see Fig. 5 ; we have used the properties $\phi_{k ; i}^{E}=-\phi_{k, i}^{E}$, and so $\phi_{k ; 1 i}^{E}=\phi_{k, 1 i}^{E}$ as well as $u_{i k}^{E}=u_{k i}^{E}$ which can be seen from (39) and (40); finally, the function $f_{i}$ is defined as

$$
f_{i}=\phi_{, 1}^{E} n_{i}-u_{i}^{E} n_{1} .
$$

If the Euler flow is assumed up to a boundary layer of negligible thickness over the body surface $\partial \Sigma_{0}$, then as the normal velocity is zero, the velocity is represented by a distribution of eulerlets $u_{k i}$ over the body surface with strength $f_{i}$

$$
u_{k}^{E}=\int_{\partial \Sigma_{0}} f_{i} u_{k i}^{E} d \sigma^{\prime}
$$

Also, on the matched boundary and in the far-field, the velocity is given by the same equation(64) such that the eulerlets are replaced by oseenlets.

\section{B. Euler wake velocity}

The Euler wake velocity is therefore given by

$$
w_{k}^{E}=\int_{\partial \Sigma_{0}} f_{i} w_{k i}^{E} d \sigma^{\prime} .
$$




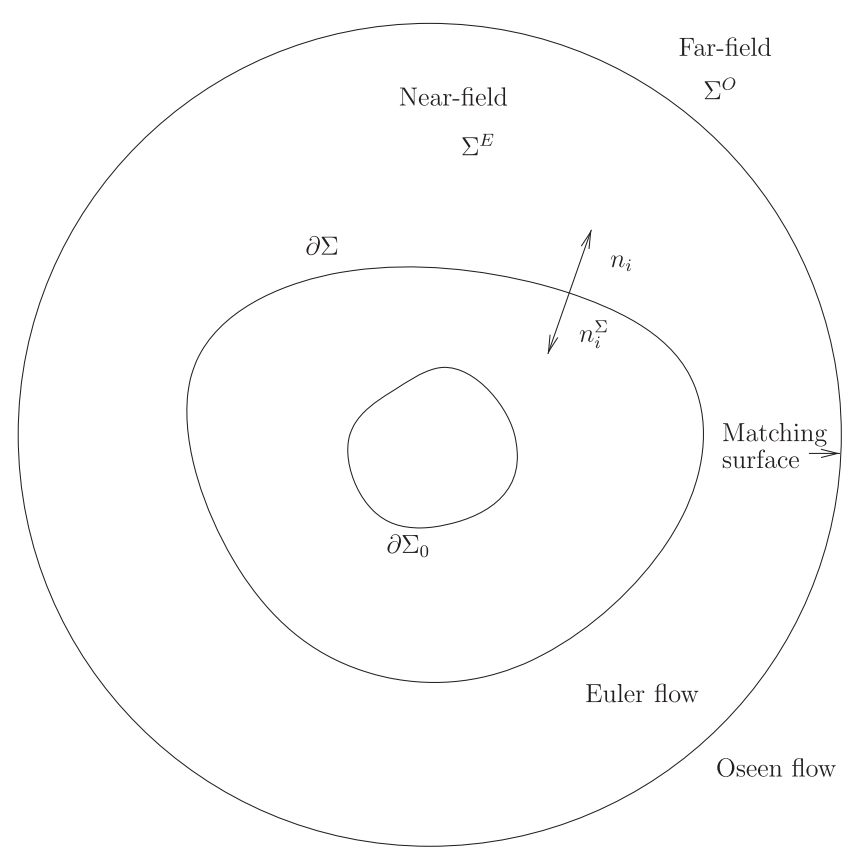

FIG. 5. The denotation of the space and the boundaries.

From Sec. IV B, the eulerlet vorticity away from its origin is zero. Therefore, taking the curl of (65) will also be zero since it is a linear integral distribution of eulerlets. Such a wake representation of wake eulerlets is unstable as in the limit, there is a different inflow along each wake line giving rise to shear. This is also found from the experiment; see, for example, Fig. 8 where the flow becomes unstable in the downstream wake after a few radius distances.

Similarly, an implication of the results in Sec. IV B is that $w_{i k, 1}^{E}=0$. Consequently,

$$
w_{i, 1}^{E}=0 .
$$

This means that the wake profile at a constant $x_{1}$ cross section will be the same regardless of value of $x_{1}$, so it remains unchanged. This is expected from the matching requirement given in Sec. IV G.

\section{Euler force}

The force representation in the Oseen flow is first given from Ref. 1, and from this, the representation in the Euler flow is obtained. The force integral in the Oseen flow is

$$
\begin{aligned}
F_{i} & =\int_{\partial \Sigma^{O}}\left[\tau_{i j}^{O}-u_{j}^{O} u_{i}^{O}\right] n_{j} d \sigma \\
& =\int_{\partial \Sigma^{O}}\left[-p^{O} \delta_{i j}+\frac{1}{R e} u_{i, j}^{O}-\left(\delta_{j 1}+u_{j}^{O}\right)\left(\delta_{i 1}+u_{i}^{O}\right)\right] n_{j} d \sigma \\
& =\int_{\partial \Sigma^{O}} \phi_{, 1}^{O} n_{i}-u_{i}^{O} n_{1}+\frac{1}{R e} u_{i, j}^{O} n_{j} d \sigma .
\end{aligned}
$$

In the limit as $R e \rightarrow \infty$, we approach the matching boundary $\Sigma^{M}$ such that $\partial \Sigma^{O} \rightarrow \partial \Sigma^{M}$ and $u_{i}^{O} \rightarrow u_{i}^{E}$, and so

$$
F_{i}=\int_{\partial \Sigma^{M}} \phi_{, 1}^{E} n_{i}-u_{i}^{E} n_{1} d \sigma
$$

From (63), then this gives a force integration in the near-field

$$
F_{i}=\int_{\partial \Sigma^{E}} f_{i} d \sigma
$$

for the force per unit boundary of the space $f_{i}=\phi_{, 1}^{E} n_{i}-u_{i}^{E} n_{1}$.

\section{ATTACHED POTENTIAL FLOW REPRESENTATIONS}

From (64), the velocity is represented by a summation of the potential flow velocity and the wake flow $u_{i}^{E}=\phi_{, i}^{E}+w_{i}^{E}$ such that

$$
u_{k}^{E}=\phi_{, k}^{E}+w_{k}^{E}=\int_{\partial \Sigma_{0}} f_{i}\left(\phi_{i, k}^{E}+w_{k i}^{E}\right) d \sigma^{\prime},
$$

where we let

$$
\phi^{E}=\int_{\partial \Sigma_{0}} f_{i} \phi_{i}^{E} d \sigma^{\prime}
$$

and

$$
w_{k}^{E}=\int_{\partial \Sigma_{0}} f_{i} w_{k i}^{E} d \sigma^{\prime} .
$$

From (63), then $f_{1}=\phi_{, 1}^{E} n_{1}-\phi_{, 1}^{E} n_{1}-w_{1}^{E} n_{1}=-w_{1}^{E} n_{1}$. However, since $w_{k}^{E}$ is an integration of the wake eulerlets $w_{k i}^{E}$ and the inflow must balance outflow, then $\int_{\partial \Sigma} \phi_{, i}^{E} n_{i} d \sigma=-\int_{\partial \Sigma} w_{1}^{E} n_{1} d \sigma$ for any domain boundary $\partial \Sigma$ enclosing domain $\Sigma$. So $\phi_{, i}^{E} n_{i}=-w_{1}^{E} n_{1}$, and

$$
\begin{aligned}
& f_{1}=\phi_{, i}^{E} n_{i}=\nabla \phi^{E} \cdot \mathbf{n}, \\
& f_{2}=\varepsilon_{3 i j} \phi_{, i}^{E} n_{j}=\left[\nabla \phi^{E} \times \mathbf{n}\right]_{3}, \\
& f_{3}=-\varepsilon_{2 i j} \phi_{, i}^{E} n_{j}=\left[-\nabla \phi^{E} \times \mathbf{n}\right]_{2},
\end{aligned}
$$

where $\times$ is the cross product. Both (71) and (72) are required in the calculation of the force $f_{i}$ on the body. Considering only the potential (71) gives only the contribution to the force from the potential, which from Table I is seen to be zero for the drag problem (giving rise to D'Alembert's paradox) and a half for the 3-D lifting problem (giving rise to Chadwick's lift discrepancy ${ }^{9}$ ). Additionally, considering (72) gives as well the contribution to the force from the wake velocity, which from Table I is seen to be all the drag (resolving D'Alembert's paradox) and an additional half for the 3-D lifting problem (resolving Chadwick's lift discrepancy ${ }^{9}$ ).

\section{A. Uniform attached flow past an aerofoil at small angle of attack}

Let us consider attached flow such that a wake line emerges at the trailing edge, so (72) reduces to

$$
w_{1}^{E}=F_{1} w_{11}^{E}=D H\left(x_{1}\right) \delta\left(x_{2}\right)
$$

for two-dimensional flow, where $F_{1}=D$ is the drag and is assumed nonzero. This represents a streamline emerging from the trailing edge in the downstream wake, which is equivalent to imposing the Kutta condition that there is a stagnation point at the trailing edge. Equation (71) represents the flow by a distribution of point sources $\phi_{1}^{E}$ and point vortices $\phi_{2}^{E}$. However, for a thin aerofoil, the outflow strength $f_{1}$ of the point sources is much smaller than the circulation strength $f_{2}$ of the point vortices. In particular, the ratio of the strength is of the order of the thinness parameter of the aerofoil (defining thinness $=$ thickness/chord). So (71) approximates to 


$$
\phi^{E}=\int_{\partial \Sigma_{0}}-\frac{\gamma \theta}{2 \pi} d \sigma^{\prime}
$$

where the potential part of the two-dimensional lift eulerlet (39) giving $-\frac{\theta}{2 \pi}$ represents a clockwise circulation point vortex on $\partial \Sigma_{0}$ with circulation strength $\gamma=\phi_{, 1}^{E} n_{2}-\phi_{, 2}^{E} n_{1}$. Satisfying the impermeability boundary condition $u_{i}^{E} n_{i}=-U n_{1}$ on the body surface where $u_{i}^{E}=\phi_{, i}^{E}$ as well as imposing the condition that there is a streamline emerging at the trailing edge (the Kutta condition) then gives a unique solution and is equivalent to the classical thin aerofoil theory representations.

\section{B. Uniform attached flow past a wing at small angle of attack}

For three-dimensional attached flow, the wake is now along a wake sheet and emerges at the trailing edge. The potential part of the lift eulerlet $\phi_{2}^{E}$ in three-dimensional flow is the infinitesimal horseshoe vortex, and from Ref. 8, it is shown that numerical discretizations of these will give distributions of horseshoe vortices; see Fig. 6. For example, from (71) and following Ref. 8, a constant spanwise distribution of lift eulerlets with strength $\Gamma$ from $x_{3}=0$ to span $x_{3}=s$ will give a contribution to the velocity potential of

$$
\begin{aligned}
\phi_{, i}^{E} & =\frac{\Gamma}{4 \pi} \int_{0}^{s}\left[\ln \left(R^{\prime}-x_{1}\right)\right]_{i 2} d x_{3}^{\prime} \\
& =-\frac{\Gamma}{4 \pi} \int_{0}^{s} \int_{0}^{\infty}\left(\frac{1}{R^{\prime \prime}}\right),{ }_{i 2} d x_{1}^{\prime} d x_{3}^{\prime} \\
& =-\frac{\Gamma}{4 \pi} \int_{0}^{s} \int_{0}^{\infty}\left[\left(\frac{1}{R^{\prime \prime}}\right), i 2-\delta_{i 2}\left(\frac{1}{R^{\prime \prime}}\right), j j\right] d x_{1}^{\prime} d x_{3}^{\prime} \\
& =-\frac{\Gamma}{4 \pi} \int_{0}^{s} \int_{0}^{\infty}\left(\delta_{i m} \delta_{j 2}-\delta_{j m} \delta_{i 2}\right)\left(\frac{1}{R^{\prime \prime}}\right), m j d x_{1}^{\prime} d x_{3}^{\prime} \\
& =\frac{\Gamma}{4 \pi} \int_{0}^{s} \int_{0}^{\infty} \varepsilon_{q i j} \varepsilon_{q m 2}\left(\frac{1}{R^{\prime \prime}}\right), j ; m \\
& =\frac{\Gamma}{4 \pi} \int_{C_{h}}^{\prime} \varepsilon_{q i j} \varepsilon_{q m 2}^{\prime} n_{m}\left(\frac{1}{R^{\prime \prime}}\right), j d l^{\prime} \\
& =\frac{\Gamma}{4 \pi} \int_{C_{h}} \varepsilon_{i j q}\left(\frac{1}{R^{\prime \prime}}\right), j t_{q} d l^{\prime},
\end{aligned}
$$

where $R^{\prime}=\left\{x_{1}^{2}+x_{2}^{2}+\left(x_{3}-x_{3}^{\prime}\right)^{2}\right\}^{1 / 2}, R^{\prime \prime}=\left\{\left(x_{1}-x_{1}^{\prime}\right)^{2}+x_{2}^{2}+\right.$ $\left.\left(x_{3}-x_{3}^{\prime}\right)^{2}\right\}^{1 / 2}$, and where $C_{h}$ is the horseshoe described by the lines around the perimeter of the area integral, given in Fig. 6. Here, $d l^{\prime}$ is an element of length of the horseshoe, $n_{m}$ is the outward pointing

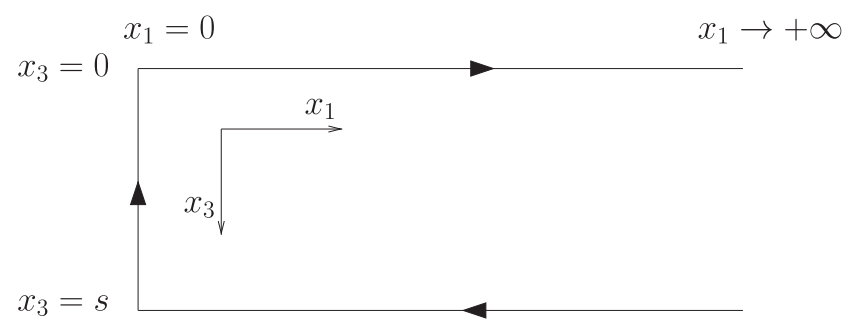

FIG. 6. Horseshoe vortex. normal to the area integral, and $t_{q}$ is the tangent vector in the direction denoted in Fig. 6. This is the Biot-Savart law integral used to determine the velocity induced by a vortex line. This discretization by horseshoe vortices can be assembled over the body surface to give a panel method or over the wing planform area to give a vortex lattice method. So with the appropriate numerical discretization, (71) reduces to the standard thin aerofoil, thin wing, and vortex lattice methods. However, the horseshoe vortex was obtained using a piecewise constant numerical discretization, but (71) now gives the possibility of considering different higher-order approximations for greater accuracy.

\section{Uniform flow past a slender body}

Furthermore, a line distribution of the potential part of the lift eulerlet is consistent with the Oseen slender body theory ${ }^{5}$ in the limit as $R e \rightarrow \infty$. In this case, an axial distribution of lift eulerlets gives a leading order distribution of two-dimensional dipoles in the crosssectional transverse flow plane. So, following Ref. 5, we have

$$
\phi^{E}=\int_{0}^{1} l\left(x_{1}^{\prime}\right)\left[\ln \left(R^{\prime}-x_{11}^{\prime}\right)\right], 2 d x_{1}^{\prime}
$$

where $l\left(x_{1}\right)$ is the lift distribution along the axis, $R^{\prime}=\left[x_{11}^{\prime 2}+x_{2}^{2}+x_{3}^{2}\right]^{1 / 2}$, $x_{11}^{\prime}=x_{1}-x_{1}^{\prime}, r^{*}=\left[x_{2}^{2}+x_{3}^{2}\right]^{1 / 2}$, and the body is positioned on the $x_{1}$ axis from $0 \leq x_{1} \leq 1$. Following Ref. 5 and using standard slender body approximations, this gives for $x_{11}^{\prime}>0$

$$
\begin{aligned}
R^{\prime}-x_{11}^{\prime} & =\left[x_{11}^{\prime 2}+r^{* 2}\right]^{1 / 2}-x_{11}^{\prime}=x_{11}^{\prime}\left[1+r^{* 2} / x_{11}^{\prime 2}\right]^{1 / 2}-x_{11}^{\prime} \\
& \sim r^{* 2} / 2 x_{11}^{\prime}
\end{aligned}
$$

and for $x_{11}^{\prime}<0$

$$
\begin{aligned}
R^{\prime}-x_{11}^{\prime} & =\left[x_{11}^{\prime 2}+r^{* 2}\right]^{1 / 2}-x_{11}^{\prime}=-x_{11}^{\prime}\left[1+r^{* 2} / x_{11}^{\prime 2}\right]^{1 / 2}-x_{11}^{\prime} \\
& \sim-2 x_{11}^{\prime} .
\end{aligned}
$$

Substituting into (77) then gives

$$
\begin{aligned}
\phi^{E} & \sim \int_{0}^{x_{1}} l\left(x_{1}^{\prime}\right)\left[\ln \left(r^{* 2} / 2 x_{11}^{\prime}\right)\right], 2 d x_{1}^{\prime}+\int_{x_{1}}^{1} l\left(x_{1}^{\prime}\right)\left[\ln \left(-2 x_{11}^{\prime}\right)\right]_{2} d x_{1}^{\prime} \\
& =\int_{0}^{x_{1}} l\left(x_{1}^{\prime}\right) 2\left[\ln r^{*}\right]_{2} d x_{1}^{\prime}=2 L\left(x_{1}\right) \frac{x_{2}}{r^{* 2}} .
\end{aligned}
$$

So twice the lift at any section, $L\left(x_{1}\right)=\int_{0}^{x_{1}} l\left(x_{1}^{\prime}\right) d x_{1}^{\prime}$ is the twodimensional dipole strength in the sectional plane; as observed by experiment. $^{6}$

\section{SEPARATED FLOW PAST A CIRCULAR CYLINDER}

Consider modeling separated rather than attached flow past a circular cylinder of normalized radius 1 centered at the origin represented by a distribution of eulerlets over the cylinder boundary producing a wake distribution.

\section{A. Potential flow}

The potential is given in terms of the potential parts of the eulerlets which can then be represented by a harmonic expansion originating from the origin such that 


$$
\phi^{E}=\frac{D}{2 \pi} \ln r+\sum_{n=1}^{\infty} a_{n} \frac{\cos n \theta}{r^{n}}
$$

for some unknown coefficients $a_{n}$, from either use of the Taylor series expansion or complex potential theory, and $D$ is the drag and outflow strength.

\section{B. Euler wake}

The wake velocity is given in terms of a distribution of the wake velocity of the drag eulerlets over the cylinder boundary; each wake eulerlet singular along the infinite half-line aligned to the $x_{1}$-axis. So, this can be represented as originating from a distribution along the line $-1 \leq x_{2} \leq 1, x_{1}=0$ such that

$$
\begin{aligned}
w_{i}^{E}(\mathbf{x}) & =\int_{-1}^{1} d\left(x_{2}^{\prime}\right) w_{i 1}^{E}\left(\mathbf{x}-\mathbf{x}^{\prime}\right) d x_{2}^{\prime} \\
& =-\int_{-1}^{1} d\left(x_{2}^{\prime}\right) H\left(x_{1}\right) \delta\left(x_{2}-x_{2}^{\prime}\right) \delta_{i 1} d x_{2}^{\prime}
\end{aligned}
$$

from (39), where $d\left(x_{2}\right)$ is the drag per unit length in the $x_{2}$ direction and $d x_{2}$ is a differential element of length $x_{2}$.

From (69), the total Euler drag is $D=-\int_{\partial \Sigma} w_{1}^{E} d \sigma=$ $\int_{-1}^{1} d\left(x_{2}\right) d x_{2}$, and from (81), the outflow is $\int_{\partial \Sigma} \phi_{, i}^{E} n_{i} d \sigma=D$, and from (82), the inflow is $\int_{\partial \Sigma} w_{i}^{E} n_{i} d \sigma=-D$.

\section{Euler drag profile}

Consider a constant drag eulerlet distribution such that

$$
d\left(x_{2}\right)=\frac{D}{2}
$$

for $\left|x_{2}\right| \leq 1$ and zero otherwise. Then, the Euler drag is $D=$ $\int_{-1}^{1} d\left(x_{2}\right) d x_{2}$.

\section{Boundary condition}

Assuming a boundary layer of negligible thickness over the body, then the impermeability condition holds for the Euler velocity

$$
\begin{aligned}
u_{i}^{\dagger} n_{i} & =\left.0\right|_{r=1}, \\
\left(\delta_{i 1}+\phi_{, i}^{E}-H\left(x_{1}\right) d\left(x_{2}\right) \delta_{i 1}\right) n_{i} & =\left.0\right|_{r=1}, \\
n_{1}+\phi_{, i}^{E} n_{i}-H\left(x_{1}\right) d\left(x_{2}\right) n_{1} & =\left.0\right|_{r=1} .
\end{aligned}
$$

Substituting in the expression for the potential (81) then gives for $r=1, x_{1}<0$ :

$$
\cos \theta+\frac{D}{2 \pi}-\sum_{n=1}^{\infty} n a_{n} \cos n \theta=0
$$

and for $r=1, x_{1}>0$ :

$$
\cos \theta+\frac{D}{2 \pi}-\sum_{n=1}^{\infty} n a_{n} \cos n \theta-\frac{D}{2} \cos \theta=0 .
$$

\section{E. Coefficients $a_{n}$}

Applying the Fourier analysis integrating over the half space $\int_{0}^{\pi} d \theta$ gives

$$
\begin{aligned}
a_{1} & =1-\frac{D}{\pi} \int_{0}^{\pi / 2} \cos ^{2} \theta d \theta=1-\frac{D}{4} \\
a_{m} & =-\frac{D}{m \pi} \int_{0}^{\pi / 2} \cos m \theta \cos \theta d \theta \\
& =-\frac{D}{2 m \pi}\left[\frac{1}{m+1} \sin (m+1) \theta+\frac{1}{m-1} \sin (m-1) \theta\right] \\
& =\frac{D}{m\left(m^{2}-1\right) \pi} \cos m \pi / 2 \\
& =\frac{(-1)^{m / 2} D}{m\left(m^{2}-1\right) \pi} \text { for } m \text { even, and } \\
& =0 \text { for } m \text { odd. }
\end{aligned}
$$

\section{RESULTS}

\section{A. Pressure distribution around the circular cylinder}

We define the pressure $p^{E}$ as an integration over the cylinder starting from the forward stagnation point on the cylinder and then integrate around the circle circumference to the desired point. So

$$
\begin{aligned}
p^{E} & =1 / 2+\int_{0}^{l} \frac{\partial p^{E}}{\partial l^{\prime}} d l^{\prime}=1 / 2-\int_{\pi}^{\theta} t_{i} p_{,}^{E} d \theta \\
& =1 / 2+\int_{\pi}^{\theta} t_{i}\left\{u_{j}^{E} u_{i, j}^{E}+u_{i, 1}^{E}\right\} d \theta,
\end{aligned}
$$

where $t_{i}$ is the clockwise tangent $\left(t_{1}, t_{2}\right)=(\sin \theta,-\cos \theta)$.

\section{B. Subcritical laminar flow}

Consider subcritical laminar flow at a Reynolds number of $R e=1.1 \times 10^{4}$. Although this flow is unsteady, let us further assume that the time-averaged mean flow can still be approximately modeled with the steady flow theory. A constant eulerlet distribution is chosen because the pressure in the wake is close to constant. Modeling the flow by a constant distribution of eulerlets in the wake domain with a wake distribution strength of 2.4 gives a pressure drag coefficient determined from (88) as 1.02 and a streamline flow given in Fig. 7. Since the wake distribution strength value 2.4 is not the same as the pressure drag coefficient 1.02, this shows that there is a contribution to the drag additional to that from the pressure drag and this is originating from the Euler wake.

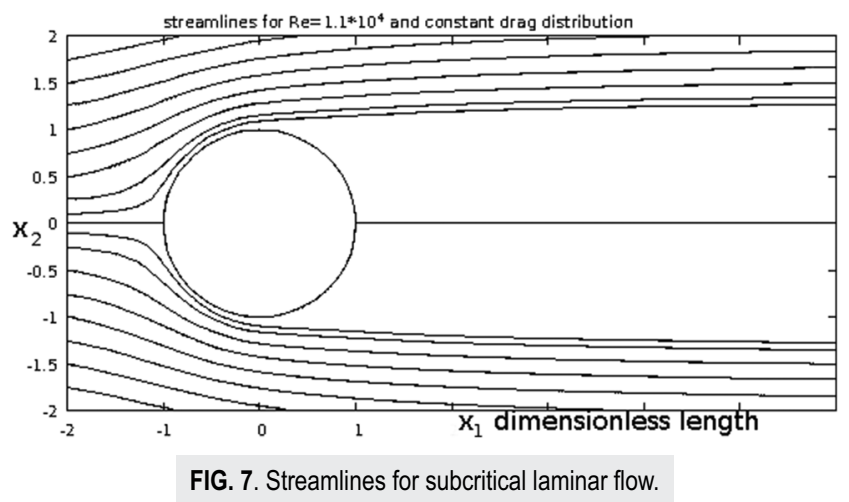




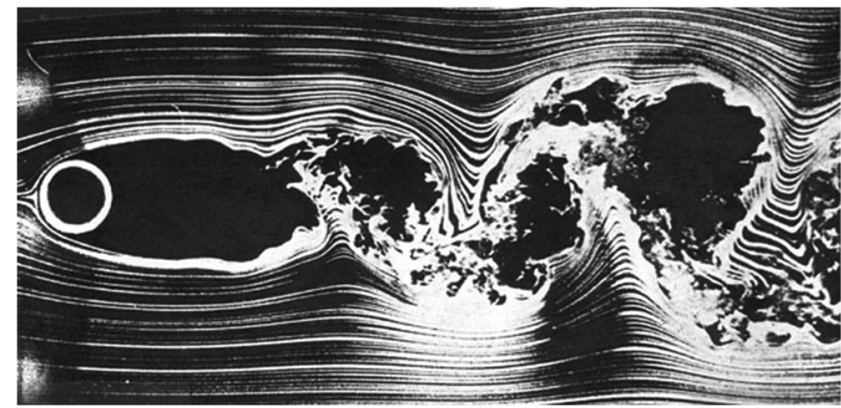

FIG. 8. Streamlines for $R e=1.0 \times 10^{4}$. [Reproduced from Album of Fluid Motion (Parabolic Press, Stanford, CA), with permission from Thomas Corke].

This compares to the flow visualization from the experiment for Reynolds number $R e=1.0 \times 10^{4}$ given in Fig. 8 . We see that the two Figs. 7 and 8 trace similar streamlines. We see that the model has correctly predicted flow separation on the fore side of the cylinder and a parabola-like separation streamline. This is in contrast to Gustaffsson's Oseen model ${ }^{20}$ which gives flow separation on the lee side and a straight separation streamline.

Using (88), the corresponding pressure distribution can be determined and is given in Fig. 9(a) and compared against the experimental pressure distribution at Reynolds number $R e=1.1 \times 10^{4}$ reproduced from the work of Batchelor. ${ }^{21}$

It is noted that characteristic features of the physics of the flow are reproduced, such as reversal in the pressure gradient at an angle of around $70-80^{\circ}$ and a negative flattened pressure profile in the wake of the cylinder.

\section{Turbulent flow}

Let us also assume that mean turbulent flow can also be approximately modeled with this steady flow theory. Again, a constant eulerlet distribution is chosen because of knowledge that the pressure in the wake is close to constant. Modeling the flow by a constant distribution of eulerlets in the wake domain but this time with a wake distribution strength of 1.5 gives a pressure drag

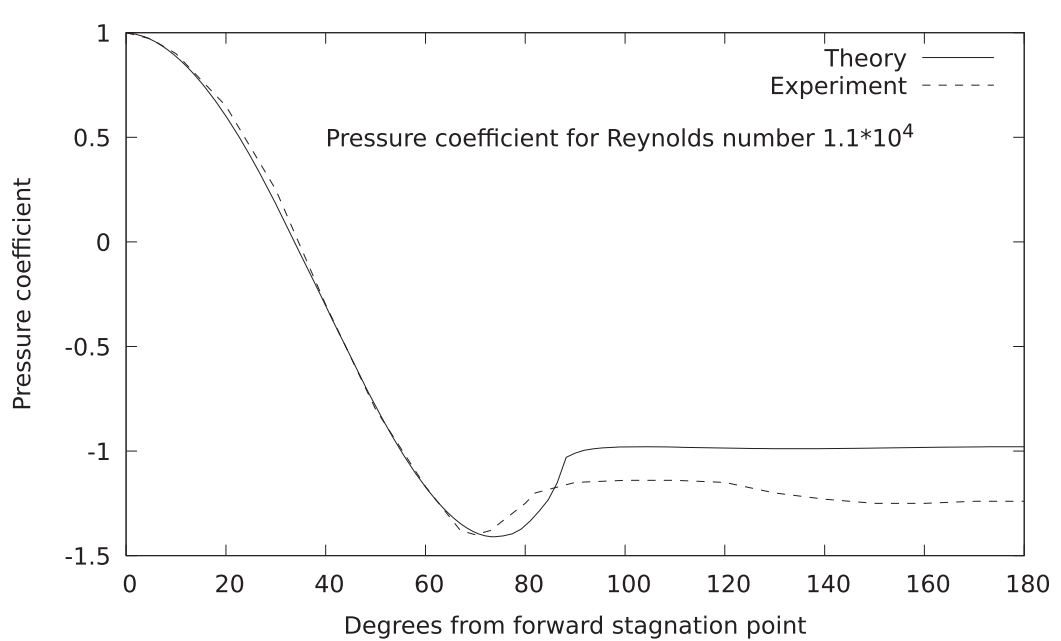

(a)

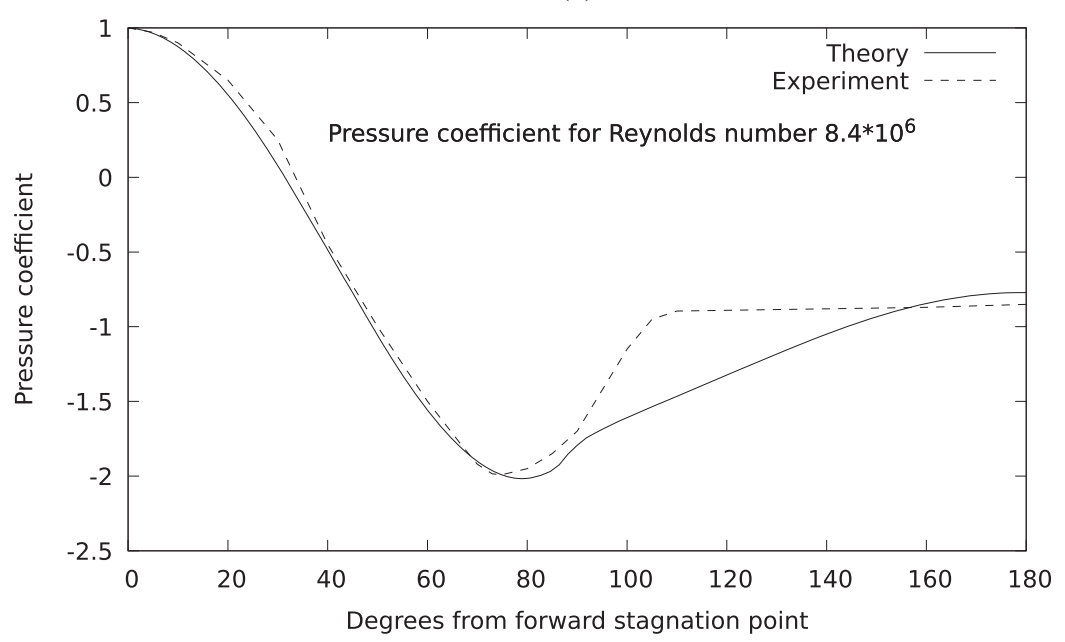

(b)
FIG. 9. Pressure distribution. (a) Pressure distribution for sub-critical laminar flow. (b) Pressure distribution for turbulent flow. 


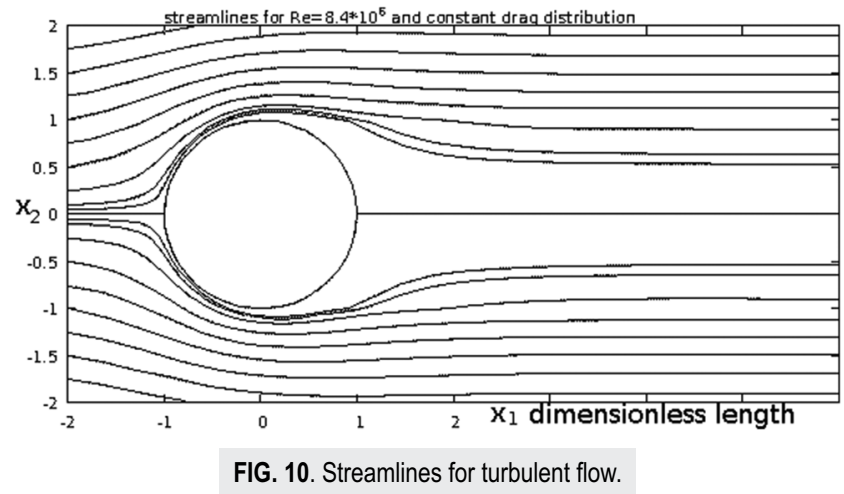

coefficient determined from (88) as 0.95 and a streamline flow given in Fig. 10.

The expected narrowing of the wake in turbulent flow is modeled; see, for example, Van Dyke's Album of Fluid Motion for the similar related case of a sphere. ${ }^{22}$ This results in a reduction in the drag coefficient; see, for example, the work of Batchelor. ${ }^{21}$ Again, it is noted that characteristic features of the physics of the flow are reproduced, such as reversal in the pressure gradient at an angle of around $70^{\circ}-80^{\circ}$ and a negative pressure profile in the wake of the cylinder; see Fig. 9(b).

\section{DISCUSSION}

A new Euler flow description is given for the velocity by a boundary integral distribution of eulerlets whose strength gives the force distribution over the boundary. So drag as well as lift is generated with the drag eulerlet describing an Euler wake with inflow balanced by a potential outflow. For potential flows, D'Alembert's paradox exists. However, this description includes an Euler wake and implicitly evaluates drag, and consequently, D'Alembert's paradox does not arise. It is also seen that this description represents an overarching representation for aerodynamic attached potential flow including thin aerofoil theory, thin wing theory, panel and vortex lattice methods, and slender body theory; the potential part of the two-dimensional lift eulerlet is the clockwise circulation point vortex, and the potential part of the three-dimensional lift eulerlet is the infinitesimal horseshoe vortex.

The theory is tested against the problem of uniform separated flow past a circular cylinder for the high Reynolds number meansteady subcritical laminar flow and mean-steady turbulent flow. The problem is uniquely described by assuming a constant strength eulerlet distribution. An analytic formulation is obtained from the Fourier series and compared to experimental results. All the flow physics is captured such as separating streamlines forward of the cylinder and pressure drop in the wake.

The pressure distribution, given in Figs. 9(a) and 9(b), follows the experiment to an acceptable level of accuracy given the approximation of steady Euler flow. The theoretical equivalence to existing attached potential flow theories as well as the close comparison to experimental results for detached flow past a circular cylinder indicates the original premise in the paper to have been appropriate. That is, to treat the Euler flow as a near-field approximation that should be matched to a far-field which is an Oseen flow in the case of a uniform flow field.

Further work will consider the application to arbitrarily shaped bodies and flows that do not have rotational symmetry, for instance, when the flow is perturbed by an ellipsoidal, arbitrarily oriented particle. For the low Reynolds number Stokes flow, this has already been conducted by Dassios and Vafeas, ${ }^{23,24}$ and so this method would then be applied for the high Reynolds number eulerlet representation given here. This would entail developing a boundary element code from this boundary integral formulation, which would have to incorporate the effect of the Euler wake generated from the eulerlet distribution.

Greater accuracy requires inclusion of the boundary layer within a Reynolds Averaged Navier Stokes (RANS) scheme, but the approach here is to investigate a fast leading order Euler method instead. This can be extended in a straightforward way for timedependent problems, and future work will be to obtain the timedependent eulerlets that can then be used to investigate flapping, swimming, and other novel propulsion mechanisms particularly for Unmanned Aerial Vehicles (UAVs) and Unmanned Underwater Vehicles (UUVs). Such problems pose great difficulty for time-dependent initial value problem computational fluid dynamics solvers. This is because of the accuracy that depletes with each time step. By contrast, it is anticipated that an Euler model using eulerlets will be more accurate because the time variation is included in the eulerlet function itself rather than from a numerical time step.

Further work will also be to experimentally test this theory. This verification will be to experimentally capture the Euler wake. For example, consider steady flow past an elliptical cylinder in a low-speed wind tunnel. We can choose cylinders with a variety of ellipticities and orientations. We will use a hot-wire probe to determine the wake velocity profile at downstream wake cross sections before the flow becomes unsteady. The probe will be controlled via a probe arm connected to a traverse mechanism capable of movements accurate to a tenth of a millimeter. The Euler wake velocity is the subtraction of the fluid velocity from the potential velocity. The theory predicts that the Euler wake velocity has an unchanging cross-sectional profile for all ellipticities and orientations, and we can test whether the experiment confirms this.

\section{REFERENCES}

${ }^{1}$ E. Chadwick, “The far field Oseen velocity expansion," Proc. R. Soc. London. Ser. A 454, 2059-2082 (1998).

${ }^{2}$ C. Oseen, Neure Methoden und Ergebnisse in der Hydrodynamik (Akad. Verlagsgesellschaft, Leipzig, 1927).

${ }^{3}$ N. Fishwick and E. Chadwick, "The evaluation of the far-field integral in the Green's function representation for steady Oseen flow," Phys. Fluids 18, 113101-1-113101-5 (2006).

${ }^{4}$ E. Chadwick, "The far-field Green's integral in Stokes flow from the boundary integral formulation," Comput. Model. Eng. Sci. 96, 177-184 (2013).

${ }^{\mathbf{5}}$ E. Chadwick, “A slender-body theory in Oseen flow," Proc. R. Soc. London. Ser. A 458, 2007-2016 (2002).

${ }^{6}$ E. Chadwick, "Experimental verification of an Oseen flow slender body theory," J. Fluid Mech. 654, 271-279 (2010).

${ }^{7}$ E. Chadwick, "A slender wing theory in potential flow," Proc. R. Soc. A 461, 415-432 (2005).

${ }^{8}$ E. Chadwick, “The vortex line in steady, incompressible Oseen flow," Proc. R. Soc. A 462, 391-401 (2006). 
${ }^{9}$ E. Chadwick and A. Hatam, "The physical interpretation of the lift discrepancy in Lanchester-Prandtl lifting wing theory for Euler flow, leading to the proposal of an alternative model in Oseen flow," Proc. R. Soc. A 463, 2257-2275 (2007).

${ }^{10} \mathrm{E}$. Chadwick and A. Kapoulas, "Using eulerlets to give a boundary integral formulation in Euler flow and discussion on applications," Comput. Model. Eng. Sci. 102, 331-343 (2015).

${ }^{11}$ E. Chadwick, "Advances in boundary element and meshless techniques XVI," in Modelling Steady Flow Past a Two-Dimensional Bluff Body by Using Eulerlets, edited by V. Mantic, A. Saez, and M. Aliabadi (EC Ltd., Eastleigh, UK, 2015), pp. 239-247.

${ }^{12}$ E. Chadwick, J. Christian, and K. Chalasani, "Using Eulerlets to model steady uniform flow past a circular cylinder," Eur. J. Comput. Mech. 27, 1550960 (2018).

${ }^{13} \mathrm{~T}$. Senguptaa and D. Patidar, "Flow past a circular cylinder executing rotary oscillation: Dimensionality of the problem," Phys. Fluids 30, 093602 (2018).

${ }^{14} \mathrm{H}$. Jiang, L. Cheng, F. Tong, S. Draper, and H. An, "Stable state of mode A for flow past a circular cylinder," Phys. Fluids 28, 104103-1-104103-13 (2016).

${ }^{15} \mathrm{G}$. Vasconcelos and M. Moura, "Vortex motion around a circular cylinder above a plane," Phys. Fluids 29, 083603 (2017).
${ }^{16}$ C. Morton, S. Yarusevych, and F. Scarano, "A tomographic PIV investigation of the flow development over dual step cylinders," Phys. Fluids 28, 025104 (2016).

${ }^{17} \mathrm{I}$. Sobey, Introduction to Interactive Boundary Layer Theory (Oxford University Press, Oxford, UK, 2000).

${ }^{18}$ L. Landau and E. Liftshitz, Fluid Mechanics, Volume 6 Course of Theoretical Physics (Pergamon, Oxford, 1959).

${ }^{19} \mathrm{M}$. Abramowitz and I. Stegun, Handbook of Mathematical Functions, 10th ed. (Dover, New York, 1972).

${ }^{20} \mathrm{~J}$. Gustafsson and B. Protas, "On Oseen flows for large Reynolds numbers," Theor. Comput. Fluid Dyn. 27, 665-680 (2013).

${ }^{21}$ G. Batchelor, An Introduction to Fluid Dynamics (Cambridge University Press, Cambridge, 1967).

${ }^{22}$ M. V. Dyke, An Album of Fluid Motion (Parabolic Press, Stanford, 1982).

${ }^{23}$ G. Dassios and P. Vafeas, "The 3D Happel model for complete isotropic Stokes flow," Int. J. Math. Math. Sci. 46, 2429-2441 (2004).

${ }^{24}$ P. Vafeas and G. Dassios, "Stokes flow in ellipsoidal geometry," J. Math. Phys. 47, 093102 (2006). 